\title{
Challenges Surrounding the Diagnosis of Autism in Children
}

\author{
Yvette Hus (iD) \\ Osnat Segal ${ }^{2}$ \\ 'Cyprus University of Technology, \\ Department of Rehabilitation Sciences, \\ Theralab Research Collaborator, \\ Limassol, Cyprus; ${ }^{2}$ Tel Aviv University, \\ Sackler Faculty of Medicine, Department \\ of Communication Disorders, Tel-Aviv, \\ Israel
}

\begin{abstract}
This comprehensive thematic review aims to highlight and familiarize readers with the challenges and pitfalls encountered in differential diagnosis of autism spectrum disorders (ASD) in children to facilitate the process of accurate identification by stakeholders. Accordingly, articles that best answer our questions and highlight our concerns were chosen from well-established publishers with prime peer reviewed journals. Included are studies showing alternate views of the issues so as to point readers to other possibilities. ASD, a complex dynamic biological-neurodevelopmental disorder, is underscored by its heterogeneous symptomology, severity, and phenotypes - all characterized by social communication deficits and presence of restricted interests and repetitive behaviours (RRBs), the core symptoms in ASD. Language and intellectual capacities do not form ASD core symptoms although vary considerably. Accurate identification is challenging as ASD is often enmeshed with other neurodevelopmental disorders, and medical comorbidities, a situation now recognized as the rule rather than the exception in child psychiatry and developmental medicine. ASD is a disorder with varying performance and severity of symptoms over time, including unexpected loss of early skills, and lost diagnosis in some children following treatment. The review reiterates the urgency in accurate diagnosis in face of the rapid rise in ASD prevalence globally, and risk-increase in delayed or denied treatment with undesirable life-long consequences for most of the affected children. In addition, a call for change is advised to circumvent the ethical dilemma posed by the present "deficit model" in ASD diagnosis. Here, ASD prevalence is presented first, followed by emphasis on importance of accurate early diagnosis, and challenges in its accomplishment due to flaws in diagnostic instruments and other contributing factors. Next follow the required criteria for accurate identification, and its difficulties attributed to comorbid conditions, gender differences, and socio-economic and cultural influences. The conclusion includes future directions and a take away message.
\end{abstract}

Keywords: autism, missed and misdiagnosis, comorbidities, neurodevelopmental disorders

\section{Introduction}

Autism, a spectrum disorder (ASD), is now recognized as a complex dynamic condition that appears early in childhood. It is enveloped in a heterogeneous etiology, and an underlying composite of disrupted developments, poor neural connectivity in interrelated systems, and varied phenotypes attributed to complex genetics where multiple genes interact with each other and the environment. ${ }^{1}$ Its noted negative impacts span neurologic, biologic, adaptive, social, linguistic, and cognitive functions, all with a complex of symptoms and needs that change throughout the lifetime. ${ }^{2}$ Although heritable, a specific identifiable ASD gene was not found, and there is no medical marker for its presence. Consequently, ASD identification is based on the affected
Correspondence: Yvette Hus

Tel + I 5 I4 4872763

Email yhus@videotron.ca 
child's developmental history, and observations of behaviour and cognitive function ${ }^{3}$ which must meet specific criteria agreed on by experts and delineated in the American Psychiatric Association's Diagnostic and Statistical Manual of Mental Disorders- DSM-5. ${ }^{4}$ Nonetheless, ASD diagnosis is rather a complex and arduous process due largely to wide variations in symptom types and severity, and their alterations with age. ${ }^{5}$ This review aims to discuss the complexity and challenges of ASD diagnosis, and misdiagnosis effects by focusing on some contributing shortcomings of the available diagnostic criteria and instruments, and frequent presence of comorbid conditions in which it is often shrouded. In fact, disentangling ASD from the co-occurring disorders further hampers diagnosis. In addition, ASD is discussed in relation to: medical issues, Attention Deficit and Hyperactivity Disorder (ADHD), Developmental Language Disorder (DLD), Developmental Coordination Disorder (DCD), Intellectual Disability (ID), ASD gender differences, and socio-cultural influences. These factors combine to pose serious obstacles and expose underlying pitfalls encountered by clinicians and researchers when aiming to achieve accurate diagnosis.

While ASD prevalence tends to vary within and between geopolitical regions, there was an unprecedented rise in the occurrence of ASD from 1 in 2000 children in the 1970 s to 1 in 54 in recent data in some regions, notes Edelson, ${ }^{6}$ and a global average estimate of 1 in 160 children according to the World Health Organization (WHO). ${ }^{7}$ This drastic rise, postulates Edelson, ${ }^{6}$ cannot be accounted for solely by improvements in identification, but rather require simultaneous investigations of connections and interactions between individual characteristics or genetics and environmental factors for more encompassing explanations. Although ASD is commonly referred to as a neurodevelopmental disorder, attesting to its neurobiological origins are a plethora of genetic studies. These include new ultra-rare heritable gene variants (passed from parents to child), ${ }^{8}$ known heritable genes, and genetic mutations or "de novo" variants (appear in the child but not in either parent), ${ }^{9}$ along with well-documented related medical and psychiatric vulnerabilities suffered by significant numbers of children with ASD. ${ }^{10-12}$ These support the conceptualization of ASD as a BiologicalNeurodevelopmental Disorder ${ }^{13,14}$ which may occur solo but is more often co-morbid with other NDDs, medical, psychiatric, or genetic disorders. ${ }^{15}$

Soke et al, ${ }^{15}$ for example, investigated the cooccurrence of behavioural and medical conditions in 4 and 8 year olds diagnosed with ASD a decade earlier as recorded by an American autism monitoring network. A re-examination of the data more recently in a crosssectional population-based study with varied sociodemographics, revealed a large majority exhibited more than one co-occurring condition. Moreover, the comorbidity type impacted on the age at which the children were first diagnosed: eg, those with developmental regressions, cognitive disability, self-injurious behaviors, and temper tantrums tended to be first diagnosed at younger ages than those with ASD without comorbidities, while those with co-occurring ADHD, anxiety, oppositional defiant disorder, and aggressive behaviors were first evaluated for ASD at an older age compared to those with ASD without comorbidities.

The researchers point out that the presence of comorbidities not only increases phenotype heterogeneity, but also tends to disguise or alter ASD symptoms so that children are more likely to be misdiagnosed and their ASD diagnosis delayed, while those with developmental or medical comorbidities such as hearing impairment, anxiety, and epilepsy tended to lose their initial ASD diagnosis altogether. To avoid these diagnoses pitfalls, clinicians are advised to screen for the presence of co-morbidities, especially since some share core-ASD symptoms.

In addition to comorbidities' interference with diagnosis are Developmental Regressions, ie, the loss of attained skills, a phenomenon that tends to impede accurate identification in very young children, was found in groups of children aged 15-24 months. ${ }^{16,17}$ The described regressions encompassed the children's communication and language skills: their joint attention declined, they exhibited reduced emotional expression, stopped responding to their name, and some also showed motor development losses. Although regression is not a common phenomenon in early development in nonASD children, researchers found that non-ASD toddler siblings of children with ASD form a high-risk group as they too may exhibit loss of skills. ${ }^{18}$ Therefore, vigilance in obtaining accurate developmental histories, and surveilling of children who lost developmental skills, in tandem with their younger siblings is highly desirable.

\section{Accurate Early Diagnosis of ASD Essential}

Accurate diagnosis of developmental disorders in early childhood, and ASD in particular, is vital for the obvious goal of targeting timely effective treatments to minimize symptoms, to increase probability of successful adaptive, 
personal, and social growth, and ultimately for achieving independent and "satisfying" function in adulthood. ${ }^{19}$ Less obvious, but important nonetheless, is the fact that early accurate diagnosis often impacts the affected person's adult life. For example, some psychiatric diseases stem from the diagnosed early onset developmental disorders, report Burger and Lang. ${ }^{20}$ Including an early onset developmental disorder diagnosis in adult patient history is vital in treatment of their psychiatric diseases as its presence profoundly affects their personality development, predicts poorer treatment outcomes, and determines need for more appropriate treatment choices. This is especially the case in syndromes with comorbidities as its combined severity impact is significantly greater than either disorder affords, note the authors. Fusar-Poli et $\mathrm{al}^{21}$ explain that delayed or missed diagnosis of ASD in early childhood is fraught with challenges when attempting diagnosis in adulthood. Furthermore, the absence of an appropriate relevant developmental history largely underlies misdiagnosis of adults with ASD seen for the first time.

Early accurate identification was found to be associated with better prognosis, ie, receiving an accurate diagnosis at a younger age is associated with better and more functional outcomes in later life as it promotes early intervention and thereby affects prognosis. ${ }^{22,23}$ Early diagnosis and interventions implemented before age 4 (between 12 and 48 months) showed significant gains in cognition, language, and adaptive behavior. ${ }^{24-26}$ Infants, for example, who were identified between 18 and 30 months and received intervention based on developmental and applied behavioral analytic principles showed significant improvement in IQ, adaptive behavior, and Autism severity two years after diagnosis. ${ }^{25}$ Similarly, children with ASD aged 30 to 42 months who received an intensive behavioral treatment showed positive outcomes in intelligence, expressive and receptive language, daily living skills, and positive social behavior two years later. Parents' reports, however, did not show a significant decrease in children's behavior problems or autistic behaviors. ${ }^{27}$ Importantly, following early identification and treatment, younger children with ASD, aged 18-48 months, achieved greater verbal developmental gains compared to those aged 48 62 months. ASD severity did not improve in either group, although non-verbal intelligence and adaptive behavior did improve in both groups. ${ }^{26}$ Overall, accumulated evidence suggests that early diagnosis and intervention are imperative in the long-term trajectories and quality of life for children with $\mathrm{ASD}{ }^{28}$
It should be noted that there is some evidence that about $9 \%$ of children that were diagnosed with ASD in early childhood may not meet diagnostic criteria for ASD by young adulthood. Youth who no longer meet criteria for ASD are more likely to have participated in earlier intervention facilities, to have a history of higher cognitive skills at age 2, and to have demonstrated a decrease in their repetitive behaviors over time. ${ }^{29,30}$ Children diagnosed at 30 months or younger are more likely to experience a change in classification from ASD to non-ASD than children diagnosed at 31 months or older. Thus, earlier identification of ASD symptoms may be associated with more responsiveness to intervention efforts or increased likelihood for over diagnosis. ${ }^{31}$

The phenomenon of loss of ASD diagnosis is referred to in the literature synonymously as recovery, resolved, loss of diagnosis, or optimal outcome. In a Turkish longitudinal study, ${ }^{32}$ a group of 39 children from well-educated upper-middle class families were diagnosed with ASD at the mean age of 2.39, and optimal outcomes were seen by mean age 5.11. In addition to early intensive intervention in behavioural programs, about $60 \%$ of the children received medication for an additional psychiatric diagnosis of either ADHD, anxiety disorder, or a tic disorder, throughout the study period. Despite the comprehensive intervention, only 9 met the optimal outcome before age 4 , while 30 achieved an optimal outcome between ages 4-10. Although some continued to need psychiatric follow-up for their co-morbid psychiatric condition, all children showed improved communication and language. In this study, the initial severity of ASD seemed to be a contributing factor to the age in which the optimal outcome occurred. The children's loss of the ASD diagnosis was attributed to their High IQ, and the development of communicative and language skills at an early age.

Similarly, an American study ${ }^{33}$ based on chart reviews of 569 children with an ASD diagnosis from age 2, who were receiving early intervention for developmental disabilities at an inner city university affiliated developmental clinic, showed 38 children's ASD was resolved by age 6.4 years. Although they exhibited cognitive gains, $92 \%$ continued to show symptoms of emotional, behavioral, and learning disabilities, and $74 \%$ continued to require educational support. Why such disparity? The Turkish study centered on families with high levels of education and upper-middle SES membership, and so were able to provide intensive support to complement the clinic and school efforts. Additionally, the co-occurring psychiatric 
disorders were diagnosed early and treated throughout the children's intervention period and beyond. In the American study, the comorbidities remained undiagnosed until school age. More importantly, the study focused on inner city children and families with known social and socio-economic disadvantages, and extra burdens not usually experienced by middle-upper strata families. Admittedly, in both situations, families struggled with the formidable challenges posed by caring for children with ASD and worrying about their futures. Apparently, the phenomenon of loss of ASD diagnosis in small groups of children has long been noted by researchers, ${ }^{33}$ but generally ASD appears to be a life-long condition for many. More research is needed to better understand the optimal outcome phenomenon, and explore how it can be extended to larger groups.

\section{Challenges in ASD Identification}

Timely and accurate ASD diagnosis in early childhood, although obviously desirable, is likewise marked with challenges, some of which are due to flaws in diagnostic criteria requirements and standardized assessment tools of the moment. Mottron, ${ }^{34}$ for example, views these as contributing factors to the extensive heterogeneity in autism, unexpected prevalence increases, and generally hampering research progress. Frith ${ }^{35}$ agrees that DSM-5 criteria hamper research, especially in recruiting participants to studies, and the "elastic" conceptualization of the autism spectrum that "blurs boundaries" between deficits, a natural variant in development, and disorders, a "glitch in neurology".

Prizant $^{36}$ notes that the diagnostic criteria for identifying ASD prior to the DSM-5, and the impetus for criteria changes, was the misdiagnosis of autism in large numbers of children- "those receiving an autism diagnosis without meeting diagnostic criteria". This contributed dramatically to the increase in autism prevalence within a short span. He based this conclusion on his extensive clinical experience and observations in many educational and rehabilitation settings, and the assessment and treatment instruments (grounded in theoretical and empirical studies), he developed with a multidisciplinary team of experts to identify young children with autism. ${ }^{37}$ The applied assessment and treatment model focuses on core challenges in ASD (SCERTS: Social Communication, Self Regulation, and Transactional Support). Prizant uncovered major factors in misdiagnosis: the diagnostic setting, presence of the parent/caregiver during the assessment, and inexperienced or "poorly" trained staff. These affect the child's behaviours, notes Prizant, so they may mimic core symptoms of autism, thereby increasing the misdiagnosis possibility. Diagnosis goal types are also possible contributors to misdiagnosis, suggests Prizant: the clinical diagnosis seeks to differentiate autism from other developmental issues, and is usually performed by appropriately trained staff using DSM-5 standards with minimal or low risk of misdiagnosis. In contrast, the educational diagnosis, performed in educational settings with government or "state" authored criteria to decide on state services eligibility, and the political diagnosis - where a child who does not meet the full criteria is misdiagnosed with autism to garner eligibility for specialized programming - contribute to the incidence of misdiagnosis.

Why is this a serious issue? Prizant explains: firstly, receiving an autism diagnosis profoundly impacts the life of the entire family. Secondly, while misdiagnosed children, mostly those with remediable communication and language challenges do benefit from services, they displace those who are genuinely on the autism spectrum. Consequently, the misdiagnosed children's "recovery", states Prizant, further propagates the "myth" that autism is "curable" rather than accept the evidence-based view that it is a life-long condition and merits support along the affected individuals' entire life-span (see Mukaddes et al, $2014 ;^{32}$ Shulman et al, $2019^{33}$ for an alternate view of curability of Autism, noted in Accurate Early Diagnosis Essential at end of section). Prizant recognizes that inevitably, there are other factors which may contribute to misdiagnosis of autism.

\section{DSM-5 ASD Classification Criteria Issues} According to the DSM-5, an accurate diagnosis of ASD hinges on the presence of symptoms from two categories: social communication, and restricted repetitive behaviours. The importance of adequacy of clinical tools for diagnosing ASD early and accurately cannot be overstated. Missed diagnosis, or a false-negative result, deprives the child from the benefits of early intervention and the chance to minimize clinical symptoms, while a false-positive result or misdiagnosing a child without the condition as having it, leads to unnecessary heartache and trouble for the child and family, and increases health services cost. ${ }^{38}$ The complexity of the autism syndrome and its wide phenotype diversity renders the process of accurate diagnosis challenging, especially in early development. ${ }^{39}$ In fact, the accurate and timely diagnosis of ASD is further 
hampered by the policies and practices of regions that limit who should diagnose, and insist that "only team approaches" be used, explain Brian et al. ${ }^{40}$ In light of the rise in autism incidence, these are inefficient as they contribute to significant delays of diagnosis and consequent treatment, and increase the possibility of misdiagnosis. The authors recommend several approaches to increase diagnosis efficiency, and note:

Regardless of the approach taken, open communication, collaboration, and consent to share information among professionals may help to achieve diagnostic accuracy and avoid duplication of effort. (p. 444)

Adding to the ASD diagnostic complication, are the DSM5 ASD classification and criteria which, in essence, harbor the potential for misdiagnosis. The DSM-5 criteria are accompanied by various stipulations noted in the general guidelines, ${ }^{41} \mathrm{eg}$, a criterion requires more than one exemplar from different contexts, or the same exemplar cannot be used to satisfy two criteria, and many more. In addition, notes Carpenter, ${ }^{41}$ the DSM-5 fails to account for behaviours and symptoms that are known to characterize ASD, and so may lead to missed diagnosis or misdiagnosis, eg, issues with play/imagination, shyness or social anxiety, language and failure to meet developmental milestones, behavioral problems and/or temper tantrums, and some poor imitation skills. In retrospect, the criteria may be more easily applied to severe ASD cases, but significantly more difficult to meet in those with a milder ASD condition where criterion behaviours are less frequent or severe, or may even be masked.

\section{Clinical Judgement and Diagnostic Tools}

The ASD diagnosis process employs an approach known as Clinical Judgement which consists of a thorough appraisal of the child's behaviour and development, and the use of standardized observations and interviews meant to help define behaviours in a consistent manner, and in essence relies on clinicians' expertise. The two instruments most often researched and used are Autism Diagnostic Observation Schedule (ADOS), based on professional observations in several settings, and a parental interview using the Autism Diagnostic Interview-Revised (ADIR). ${ }^{42}$ Both tools, with high sensitivity and specificity in diagnostic accuracy, are considered "Gold Standard". Nonetheless, accurate diagnosis was difficult to achieve when only one suggested an ASD diagnosis, and a standard approach to resolution of the disparate results was unavailable. ${ }^{42}$

Efforts were made to correct these difficulties in a large multi-site Study of Early Childhood Development (SEED), spanning six states. The study was led by researchers from the CDC (US Centres for Disease Control and Prevention), and reported by Wiggins et al. ${ }^{42}$ The SEED participants were recruited from one of the three determining groups: (1) the general population, (2) children with assorted developmental delays, and (3) children with ASD. In total, 2600 children were assessed using standard tools including ADOS and ADI-R to classify ASD or non-ASD groups. Consequently, diagnostic algorithms were altered for ADOS and the ADI-R social, communication, and behavioural domains. The altered algorithms provided balanced specificity and sensitivity results and succeeded in revealing ASD missed and misdiagnosis in groups of children, and so demonstrating more diagnostic accuracy, while the resulting classification successfully resolved disparate outcomes. The success of SEED resides in the fact that the operationalized clinical judgment approach with an embedded solution to conflicting results provides a consistent diagnostic plateau, instead of relying on disparate individual clinician expertise. This increases the odds for accurate ASD diagnosis, and serves as a base for clinical agreements. The altered algorithms, therefore, constituted an important advancement in ASD identification for both clinicians and researchers.

Attempts at using self-reports for ASD screening largely yielded poor results. For example, self-report questionnaires for diagnosing ASD in adults were suggested in the past for prioritizing individuals who need specialist assessment. In particular, the short version of the Autism Spectrum Quotient (AQ-10) questionnaire was recommended as a referral tool. ${ }^{43}$ However, following findings of a high rate of $64 \%$ false-negative misdiagnosis, ie, patients with ASD categorized as not having ASD by the AQ- $10,{ }^{44}$ this recommendation was rescinded, and it is no longer recommended as a stand-alone referral tool (NICE, 2021). The example highlights the risks in using selfreport questionnaires without a complement of clinical assessments. Additionally, a study ${ }^{45}$ compared the Ritvo Autism Asperger Diagnostic Scale (RAADS-R) self-report with the short AQ-28 and AQ-10 in ability to correctly categorize ASD or non-ASD in referred individuals. It demonstrated the tools have disparate and weak diagnostic power: the RAADS-R sensitivity or true positive rate was $73 \%$, ie, it correctly identified ASD in $73 \%$ of cases, but 
missed $27 \%$. The AQ-28 specificity or true negative rate was $70 \%$, ie, while $70 \%$ were correctly identified as nonASD, 30\% were misdiagnosed as having the condition. The authors concluded that none of these instruments have sufficient validity to reliably predict a diagnosis of ASD in outpatient settings. ${ }^{45}$

Recently, self-reported autistic traits were assessed in adult individuals with ASD, schizophrenia, spectrum disorders (SSD), and non-clinical controls (NCC), ${ }^{46}$ using the long version AQ 50-item questionnaire. The ASD sample scores were significantly higher than those in the NCC. Interestingly, no significant differences were detected between individuals with ASD and SSD. These findings raise concerns regarding the validity of $\mathrm{AQ}$ as the only screening tool in clinical populations. In all, the present findings suggest that self-reporting questionnaires are insufficient on their own to make a clinical determination, and therefore should be used as a complement rather than a replacement of clinical assessment in the screening process.

\section{ASD and Restricted Repetitive Interests and Behaviours (RRBs)}

The core deficits in all ASD phenotypes are in social communication, and the presence of RRBs stipulated in the DSM-5 as an obligatory symptom in order to receive an ASD diagnosis. Detecting RRBs is important as they seriously impact social and communicative developments, and interfere with life activities, state Richler et al. $^{47}$ However, identifying their presence presents a challenge. Firstly, RRBs include a wide range of compulsive behaviours which may be difficult to detect, and may change over time. Studies ${ }^{47}$ of longitudinal monitoring of RRBs in 2-year-old ASDdiagnosed children included subsequent exams at ages 3, 5, and 9. They identified two types of RRBs: "repetitive sensorimotor" behaviours (RSMs) - these exhibited consistent severity over time, while the "insistence on sameness" (IS) behaviours, in contrast, were inconsistent: while initially presenting as weak, their severity increased over time. Lai et al, ${ }^{48}$ for example, noted RRBS in ASD diagnosed girls' tend to centre on repetitive interests and topics (IS type behaviours) which resemble interests of non-ASD peers; consequently, they may go unnoticed and undiagnosed. Although RRBs are considered principal behaviours, the RSMs may also signal the presence of sensory processing of information difficulties. ${ }^{29}$ Therefore, when establishing an ASD profile, it is advisable to investigate children's sensory processing status as well. In addition, RRBs are often present in conditions such as Obsessive Compulsive Disorder (OCD), while in non-ASD diagnosed children with significant intellectual disabilities (ID), they occur frequently and persist throughout their life-span. ${ }^{29}$

Another complication is the uncanny similarity between Gilles de la Tourette (GTS) and $\mathrm{ASD}^{49}$ as both are syndromic NDDs occurring more in boys, and both exhibit RRBs, and vocal and verbal Tics. These are rarely diagnosed in preschool years but impulsivity and various obsessive compulsive symptoms precede while tics appear in early school years. ${ }^{50}$ Tics are defined as uncontrollable simple (eg, eye blinking) or complex body movements and sounds. Some RRBs may appear "tic-like" and difficult to differentiate from tics characteristic of GTS. Although both children with ASD and those with GTS produce vocal tics, the GTS vocal tics may consist of "barking", and their verbal tics consist of echolalia (repeating others' speech), palilalia (involuntary repeating one's own speech), or coprolalia (speech laced with obscenities). Those with ASD, however, demonstrate vocal "tics" such as humming, and bird-like chirping, while their verbal tics consist of immediate and delayed echolalia and "scripting", ie, repetition of words, phrases, or sounds from other people's speech, or from media, but do not exhibit coprolalia. However, some exhibit tics from both as the comorbidity of ASD in GTS is believed to be between $20 \%$ and $28 \%$ while GTS, predominantly a tic disorder, is frequently misdiagnosed as $\mathrm{ASD} .{ }^{50}$

In short, symptoms of obsessions, compulsive behaviors, and vocal and verbal repetitions are common in both conditions. A recent, large study ${ }^{49}$ with children and adolescents attempted to sort out the GTS phenotypes from ASD. The ADOS social aspects showed significant differences between GTS with ADHD, and OCD comorbidities from ASD; no difference was found when compared to ASD with comorbid conditions. Another study ${ }^{51}$ found that various movements such as Habits (nail biting, picking, thumb sucking, and hair twirling) and Stereotypies (eg, body rocking, tapping, hand flapping, and arm waving) may resemble tics and both may coexist in individuals, but stereotypies appear prior to age 3 and are experienced as pleasant, while tics tend to appear later, and their effects are quite aversive. 


\section{ASD and Social (Pragmatic) Communication Disorder (SPCD)}

While the DSM-5 provides criteria for determining severity levels for ASD, and requires presence of RRBs, the added category of SPCD has no assigned severity levels, and, in fact, requires the absence of RRBs as this forms an ASD differentiation variable. The added SPCD category as a distinct disorder with deficits in social verbal and nonverbal communication but without the presence of RRBs is especially problematic note researchers. ${ }^{52}$ Highlighted are the absence of validated and reliable assessment measures, and the shared symptomology with other NDDs as significant challenges for a distinct diagnosis. The researcher suggests that SPCD is best conceptualized as a dimension symptom profile spanning the NDDs that commonly co-occur with ASD, rather than a distinct entity. Furthermore, the SPCD diagnosis is based on deficits in social verbal and non-verbal communication, developments that are hinged on sufficient language levels to detect pragmatic issues, and so may not be evident prior to preschool age, and even later in those with a concomitant language disorder in the face of increased language and social demands. Other researchers ${ }^{53}$ concur: although treated as mutually exclusive, the description of social and communication deficits in SPCD are sufficiently similar to the ones delineated in ASD to cause differentiation confusion.

In fact, the validity of treating SPCD as a distinct category tends to be questioned. A large study ${ }^{54}$ with wide-age range participants (4-18 years) examined whether the SPCD symptoms aggregate to form a syndrome separate from ASD. The study failed to expose a distinct syndrome; instead, the symptoms seemed to border ASD, and revealed that while not meeting ASD criteria, individual with border results also showed a need for support. A study ${ }^{55}$ aimed at identifying differentiation features between ASD and SPCD showed ASD demonstrates more severe features; however, it failed to expose a distinct category. Instead, the SPCD features fell along a continuum. These studies have clear implications for clinicians and researchers: they illuminate the need for significantly more research to determine characteristics that define SPCD uniquely, ie, uncover phenotypes, and the urgent need to construct validated appropriate tools for accurate differentiation of SPCD from ASD and from other NDDs to determine whether, in fact, SPCD forms a distinct category.

\section{ASD and Medical Co-Morbidities}

Evidence is mounting for the association of somatic or medical conditions and developmental disorders noted in a large meta-analysis of medical comorbidity in a wideage range of children and adolescents with ADHD and in those with ASD as it revealed medical disorders in these populations seem to be ubiquitous. ${ }^{56}$ The meta-analysis showed the most frequent medical co-morbidity in those with ASD was gastro-intestinal disease (GID). An association was found between GID and sleep disorders, and in those with GID, the risk for sleep disturbance, seizures, or both was doubled. ${ }^{57}$ Frequency of GID tended to change with different studies and fluctuated between $70 \%, 46 \%$, or no significant difference from neurotypical children. In addition, $60 \%$ of the children also exhibited associated food selectivity issues. ${ }^{56}$ In a GID study ${ }^{14}$ with difficult symptoms (inflammatory bowel disease excluded) frequency was shown to be between $10 \%$ and $12 \%$. A systematic review of effectiveness of managing GID problems by restoring gut microbiota with pre- and probiotics showed limited effectiveness in either alleviating the issue or minimizing the accompanying disruptive behaviours. The study highlights the difficulties in managing GID issues in children with ASD as it seems to be resistant to treatment. ${ }^{58}$

Muskens et $\mathrm{al}^{56}$ noted that increased ASD symptom severity was associated with increased GID frequency. They point out that affected individuals with ASD who have difficulty expressing their symptoms and pain verbally tend to exhibit disruptive behaviours; these mask their medical conditions, and so their somatic conditions may remain undiagnosed. The researchers emphasize the importance of employing collaborative screening models to address both somatic and psychiatric disorders and provide personalized interventions. In fact, when multiple co-occurring medical conditions are present in the child, each additional medical condition increases the severity of behavioural impairments. ${ }^{57}$ The American Academy of Pediatrics (AAP) recognizes that co-occurring medical conditions exert additional adverse impacts on the function of the child, the families, and on the clinical management of the child. ${ }^{29}$ A recent eye-opening headline in British Columbia University's Arts and Humanities News announcing, "Mentalhealth conditions alarmingly high among children with autism"12 brought home the implications of cost and worries for families and societal agencies. A large number of individuals with ASD, including preschool 
children, were reported with comorbid mental health issues; these tended to be neglected as the focus was on their autism. Moreover, writes the reporter, ${ }^{12}$ there are not enough people trained in providing mental health treatment for those with ASD, making this a dire reality indeed.

Feroe et $\mathrm{al}^{59}$ report that to date, there are no medical treatments for the autism complex, but nonetheless a large number of physicians incorporate medication in management of ASD, mostly with ineffective results. In addition, behavioural interventions were not found appropriate for all individuals with ASD. These factors, combined with insufficient number of well-trained physicians in management of mental health in ASD make for a rather worrisome picture. To combat this situation, the $\mathrm{AAP}^{29}$ recommends that physicians incorporate medications in their management of comorbid conditions such as seizures, ADHD, anxiety, mood disorders, and disruptive behavior disorders. In all, the issues of medical comorbidities are significant as they affect the child and the entire course of intervention. It is hoped that with more effective focused management of medical problems, children's response to intervention to reduce ASD symptoms and increase their adaptive capacity will be enhanced significantly.

It is important to note that with the considerable rise in prevalence, Autism became the disorder "du jour", displacing interest in "bipolar" and "ADHD" disorders to a great extent. $^{60}$ The rise in interest is attested to by the plethora of publications on all aspects of the disorder spanning molecular, neurobiological, medical, and educational research, to name a few. In fact, interest in "medical solutions" to tackle ASD core deficits has grown. Recent pharmacological clinical trials are now exploring novel pharmacologic treatment to address the social symptoms associated with ASD. ${ }^{61}$ Since medications designed for other psychiatric disorders are largely ineffective in addressing ASD deficits, ${ }^{59}$ this is a welcome development in the efforts to combat a core problem in ASD symptomology.

\section{ASD and Comorbid Conditions in Early Childhood}

The common developmental delays in early childhood, especially in toddlers with no genetic syndromes and overt neurological or sensory impairment, usually fall into one of the following neurodevelopmental diagnoses: ASD, Attention Deficit and Hyperactivity Disorder (ADHD), Developmental Language Disorder (DLD),
Developmental Coordination Disorder (DCD), and Intellectual Disorder (ID). These diagnoses tend to share common characteristics such as language and motor delays with ASD. Thus, the presence of additional developmental delays may mask an ASD diagnosis leading to a missed or an unrecognized diagnosis of ASD on the one hand, and a misdiagnosis of a secondary neurodevelopmental condition as a primary condition, on the other hand.

In a large study ${ }^{62}$ with a cohort of 221 children, the initial diagnostic impression prior to age 6 by an intradisciplinary diagnostic team included language deficits $(70 \%)$, motor difficulties $(67 \%)$, attention problems (46\%), and cognitive difficulties (42\%). Although an ASD diagnosis was initially ruled out, it was finally acknowledged after age 6. This example epitomizes the difficulties in early differentiation diagnosis. Initially, specific symptoms traditionally associated with ASD such as atypical eye contact and reduced social behavior, also common in other conditions, ${ }^{62,63}$ may bias diagnosticians towards an ASD diagnosis. In fact, research indicates that the risk of misdiagnosis is higher in infants younger than 16 months, or prior to age $3 .^{64,65}$ However, there are cases of children younger than 24 months who have been successfully diagnosed with ASD. ${ }^{66}$ The following sections address the challenges in the identification of ASD that may lead to missed and misdiagnosis in early childhood.

\section{ASD and ADHD}

Differential diagnosis of pre-school infants, toddlers, and children with ASD and co-occurring ADHD poses a noted challenge. The criteria for ASD include difficulties in social interaction, communication, and stereotypic behavior, whereas according to the DSM-5, the ADHD criteria encompass symptoms of inattention, hyperactivity, and impulsivity. Nonetheless, $37-85 \%$ of children with $\mathrm{ASD}^{67}$ exhibit comorbid ADHD symptoms, while autisticlike symptoms and features are reported in children with ADHD. ${ }^{63,68}$ These difficulties include social cognition, language development issues, and communication difficulties. ${ }^{63,69,70}$ The co-occurrence of ADHD in children with ASD, and deficits in social cognition and language in children with ADHD make early differential diagnosis between ASD combined with ADHD, and ADHD per-se a non-trivial process.

Adding to this complexity, and posing an additional challenge to differential diagnosis in young children with ASD and ADHD, are difficulties in recognizing gaze direction and face processing including recognition of 
emotional expressions, and inverted face tasks. ${ }^{71-73}$ Difficulties recognizing gaze direction and face processing tend to lead to reduced joint attention and alter the development of social cognition. ${ }^{74}$ Both toddlers with ASD and those with ADHD may show atypical eye contact, reduced followings of the conversational partner gaze shifts, and reduced use of these for expressing communication intents such as initiations and confirmations. Since unusual eye contact in toddlers tends to be interpreted by educators and diagnosticians as a sign of reduced interest in people, it may lead to an ASD misdiagnosis, although this behavior could possibly stem from attentional difficulties (to the best of our knowledge, presently, no data is available on this type of misdiagnosis).

Furthermore, although the AAP set guidelines for diagnosing ADHD in 4 year olds, ${ }^{75}$ it is exceedingly difficult to obtain an accurate diagnosis in children younger than 7 . Differentiating transient from significant clinical behaviours of ADHD poses a veritable challenge, compounded by lack of clear diagnostic markers and tools. ${ }^{76}$ This situation increases the risk of a diagnostic bias towards ASD over ADHD, especially when additional motor and language delays are present. More studies focusing on differential diagnosis between ASD and ADHD in early childhood are clearly needed.

\section{ASD and DLD}

The core symptoms of children with ASD include impairments in communication, social interaction, and RRBs. These core symptoms differ from those of DLD, previously known as Specific Language Disorder (SLI) and also termed Language Disorder (LD), as per the DSM-5. The core symptoms of children with DLD include impairments in the ability to express and/or comprehend language with difficulties in at least one of the linguistic domains: morphology, syntax, lexicon, phonology, and pragmatics, in the presence of normal non-verbal IQ, and no other significant developmental deficits. ${ }^{77,78}$ Nevertheless, there are areas of commonalities between ASD and DLD that encumber differential diagnosis. It has been reported that $50-60 \%$ of children diagnosed with ASD have language impairment, ${ }^{79,80}$ independent of nonverbal IQ. Furthermore, high rates (about 50\%) of children who received late diagnosis ASD, first received a diagnosis of language disorder suggesting that the presence of language difficulties may lead to early missed diagnosis of ASD. ${ }^{81}$ It is therefore important to consider two types of autistic profiles when diagnosing preschool toddlers and children: 1). Autism Language Impaired (ALI), and 2). Autism Language Normal (ALN). In both profiles, pragmatic aspects of language use such as turn taking, correcting misunderstandings, or giving relevance information, ie, details pertinent to the matter at hand, are usually compromised to some degree. In ALI, however, structural linguistic domains including syntax, morphology, lexicon, and phonology might also be impaired resulting in difficulties in expressive and/or receptive language. In contrast, in ALN structural linguistic domains are intact. $^{77,82,83}$

A few hallmarks may assist in the differential diagnosis between ASD (ALN/ALI) and DLD. First, although young children with DLD and ASD show delays in interpersonal skills such as sharing affect, using gaze, and initiating communication, the ability of children with DLD to engage in pretend play, use gestures to communicate and respond to language can contribute to differentiation from ASD. ${ }^{84}$ Moreover, even though both, children with ASD and those with DLD, face difficulties in pragmatics, children with ASD also have difficulties in Theory of Mind (ToM) or the ability to make inferences about the mental states of others. In children with DLD, pragmatic difficulties are attributed to deficits in structural language ${ }^{78}$ while in children with ASD, difficulties in pragmatics are attributed to deficits in ToM. These tend to alter mentalization capabilities or the ability to use and understand linguistic information that addresses hidden intentions and motivations of others, as well as to manage social behaviors and relationships. $^{78}$

In light of this, while pragmatic abilities of children with DLD may develop in parallel to their structural language development, children with ASD may not show this developmental pattern. Instead, those with ASD may demonstrate long-lasting difficulties in pragmatic tasks that involve high-order understanding of ToM (inference about someone's belief about another's belief), ${ }^{85}$ despite intact structural language abilities. Finally, there are important differences in social play and social interaction with peers between children with DLD and those with ASD. Preschool children with DLD may show difficulties during play in turn-taking, and access behaviours to gain attention or things. However, peer interaction strengths were also reported in terms of the children's abilities to make friends, to use verbal and non-verbal behaviour to make joint decisions with peers, and to engage with peers in social pretend play. ${ }^{86}$ In contrast, social play development of children with ASD is reduced in the presence of 
reduced social motivation that often constrains the development of social play. ${ }^{87}$

\section{ASD and DCD}

ASD diagnosis is based on difficulty with social interaction, communication, and RRBs. DCD, or "dyspraxia", on the other hand, is characterized by significant difficulty performing accurate and rapid gross- and fine-motor skills, including problems with coordination, praxis, postural control, and balance at an age-appropriate level, and without known overt neurological injury. ${ }^{88}$ In theory, ASD and DCD, each involve difficulties in distinct areas of development so that symptoms characterizing each condition can be distinguished easily. In practice, however, although neither gross- nor fine-motor impairments are defining characteristics of ASD, according to the DSM-5, motor developmental delays and deficits are common in ASD. ${ }^{88,89}$

The late Dr. Leo Kanner's initial description of children with Autism suggests "somewhat clumsy in gait and gross motor performance". ${ }^{90}$ More recently, several researchers demonstrated that when compared to neurotypical (NT) peers, individuals with ASD have significant motor praxis and coordination impairments, although they may not carry a formal diagnosis of dyspraxia or DCD. ${ }^{91,92}$ Both ASD and DCD children tend to reach key motor milestones (crawling, standing, walking) later than NT peers. ${ }^{64}$ Some researches even argue that motor impairments can be considered a fundamental feature of ASD implying that no additional diagnosis of DCD is mandatory. ${ }^{93}$ Additional difficulties that are mutual to both ASD and DCD, and may further complicate differential diagnosis, include visual face processing, eg, identification of the same face with different expressions. Also noted are difficulties in social interaction, personal relationships, and coping skills, although social deficits in the DCD group may not be as marked as those seen in children with ASD. ${ }^{64}$ There is evidence indicating that deficits in nonverbal communication behaviors that typically emerge in early development, such as eye contact, facial expressions, gestures, as well as the capacity to share enjoyment and participate in simple back-and-forth games (eg, peek-a-boo), are mostly specific to ASD. ${ }^{94,95}$ However, such behaviors may also be delayed in children with DCD, especially in those with difficulties in face processing, gaze following, and imitation. ${ }^{64}$

The co-occurrence of motor deficits in ASD and the fact that motor delays may be the first sign for a developmental problem that later is diagnosed as ASD may complicate differentiation between ASD and early DCD. This is especially true in infants and toddlers under age 2, a developmental period in which other characteristics of the ASD diagnosis such as impaired social interaction and communication, and RRBs are not fully demonstrated. Moreover, dual diagnosis of ASD plus DCD may be missed because motor deficits are attributed to the ASD condition. A recent study on a large dataset $(11,814$ participants) found about $87 \%$ of the children with ASD are at risk for DCD throughout childhood and adolescence, whereas only about $15 \%$ of this sample held a dual diagnosis of ASD comorbid with DCD. ${ }^{89}$ Moreover, although to-date the DSM-5 criteria specifically allow children to hold a dual diagnosis of ASD and DCD, there is still a lack of consensus in the field about whether DCD and ASD are distinct disorders with some shared motor features, or whether some individuals with ASD have DCD while others do not. ${ }^{96}$ In the absence of norms that differentiate motor performance of ASD and DCD, clinicians may find it challenging to determine whether the motor ability of the child impacts daily functioning above and beyond the core impairments associated with ASD as required by the DSM-5; consequently, dual diagnosis is rarely given. ${ }^{96}$ This type of misdiagnosis may prevent access to appropriate treatment that can promote participation of ASD children and adolescents in sports, playground games, and other leisure and daily activities.

\section{ASD and ID}

According to Srivastava and Schwartz, ${ }^{97}$ intellectual disability (ID) is a condition characterized by below average intellectual function (IQ <70) in conjunction with significant limitations in adaptive function. The disorder may occur as an isolated phenomenon, and in some cases accompanied by facial dysmorphic features, neurological signs, sensory impairments (eg, vision, hearing), seizures, and behavioral disturbances. In ASD, in contrast, the primary difficulty is in communication but not in intellectual functioning. Nevertheless, many children with ASD also exhibit co-morbid ID. ${ }^{98}$ It has been estimated that $4-40 \%$ of individuals with ID exhibit co-occurring ASD, while $50-70 \%$ of individuals with ASD are also diagnosed with ID. ${ }^{99,100}$

Except for children with profound delays, it is difficult to differentiate ASD from those with comorbid ID in children with suspected ASD aged 2-3 or younger ${ }^{101}$ due to cooperation problems in non-verbal tasks that hamper 
assessment of non-verbal cognitive abilities. Moreover, motor and language delays may accompany both ASD and ID children in young ages, making it difficult to distinguish whether the developmental delays are part of ASD, ID or both. In 4 to 6 year olds with ASD, language and motor delays might be less severe, making differentiation easier. Language delays, for example, are reported in most 2-3 years old with ASD, but most children (81\%) closed this developmental gap either by ages $5-6^{102}$ or by age $11 .^{103}$

Assessing children with ASD compared to those with accompanying ID may be easier between ages 4 to 6 when the accompanying delays can be more easily distinguished from the core ASD characteristics, and children tend to be more compliant. Importantly, in the last decade, the trend shows reduced incidence of ID diagnosis and increased diagnosis of ASD in its lieu. This phenomenon of diagnostic re-categorization can explain, among other factors, the vast recent increase in prevalence of ASD diagnosis. It is reported, for example, that the decrease in ID prevalence was approximately $64 \%$ of the increase of autism prevalence for children aged 3-18. Consequently, individuals who were diagnosed with an ID in the past are now recategorized as individuals with ASD. ${ }^{104}$ A recent case report $^{105}$ provided an example of a nine-year-old boy from Pakistan whose school teacher regarded as autistic with learning disabilities. A careful clinical assessment, however, revealed a non-autistic diagnosis, and an appropriate adjustment to his therapeutic plan followed. This example highlights the influence of ASD misdiagnosis on treatment.

Another difficulty is differentiating primary ID without ASD from ID with ASD due to the decline in social abilities with increased ID severity. Hence, when social deficits and RRBs are observed in individuals with ID, clinicians must determine whether an additional diagnosis of ASD is necessary or whether these symptoms are part of the ID condition. A dual diagnosis has important implication for prognosis. Individuals with ID and autistic features (ID with ASD) tend to have more difficulties in functional independence and adaptive behaviors compared to individual with a diagnosis of ID only. ${ }^{106}$ They are also likely to have an underlying specific genetic etiology or a genetic syndrome. ${ }^{107}$ The rate of severe-to-profound ID is higher among ASD with ID individuals than in those with ID alone. ${ }^{108}$ Finally, services and supports afforded to individuals diagnosed with ASD are generally more comprehensive than for those with ID. This disparity motivates many parents to seek out an additional ASD diagnosis in order to secure extra services, a practice that muddles the question of when and for whom a comorbid ASD diagnosis is appropriate. ${ }^{104}$ In addition to confusing the issue, this phenomenon certainly contributes to the ever-growing incidence of ASD.

In sum, comorbidity of attention, language, motor and/ or cognitive deficits may complicate the diagnosis of ASD and affect the severity of symptoms. Nonetheless, early intervention services for improving communication as well as their whole range of developmental aspects should be provided to any child with developmental risk symptoms. Instead of adhering to a "wait-and see" approach, a provisional diagnosis can be assigned at the time the developmental concern is identified, and intervention initiated with goals based on the presenting concerns. This is with a view to stimulate development since research indicates that early and intense intervention is highly recommended to support growth in social communication, language, play, and adaptive behaviours. ${ }^{109-111}$

\section{ASD and Selective Mutism (SM)}

Although SM is not classified as a neurodevelopmental disorder, it is included here as it shares some characteristics with ASD thus may be misdiagnosed as ASD, especially when under cultural influences where "silent children" are not viewed as a problem. ${ }^{112}$ The DSM 5 (312.23) classifies $\mathrm{SM}$ as a rare disorder $(0.03 \%$ to $1 \%$ prevalence) in the broad anxiety category. The diagnostic criteria for SM include: a consistent failure to speak voluntarily in specific social situations in which there is an expectation for speaking, as in school, despite speaking at home, for example, indicating the selective failure to speak is not due to limited or lack of spoken language knowledge; the disorder impacts negatively on the affected individual's social communication, and school or occupational performance. A recent study indicated Anxiety Disorder is present in $80 \%$ of diagnosed children, even though SM is not part of the diagnostic criteria, and in $69 \%$ of these, social phobia was the most frequent comorbid condition. Furthermore, although symptoms may be present by age 4.5 , typically it is diagnosed at school age, and may persist to adulthood if left untreated. ${ }^{113}$ A review study notes that social anxiety is prominent in SM, and highlights that the common symptoms between SM, Social Anxiety, and ASD have diagnostic and treatment implications. $^{114}$ A Swedish retrospective clinical chart review of $4-18 \mathrm{yr}$ olds $^{115}$ found SM tends to occur more 
often in girls than boys (ratio 2.7:1). The study exposed ASD comorbidity in $63 \%$, and this SM+ASD group showed later symptom onset and diagnosis age, more frequent history of speech delay, many more with borderline IQ or ID, and only one gender difference: more boys from families with non-Swedish languages. The study highlights the overlap between SM, ASD, and ID, and the importance of identifying comorbid conditions when $\mathrm{SM}$ is suspected. The need for increased vigilance in differential diagnosis in disorders with overlapping symptoms cannot be over-stated.

\section{ASD Gender Differences}

The prevalence of ASD in boys is higher than ASD in girls especially in individuals with higher IQ. ${ }^{116}$ According to the CDC, 1 in 38 boys and 1 in 152 girls aged 8 were diagnosed with ASD, suggesting that the diagnosis ratio of males versus females is about $4: 1$. Also, among children identified with ASD, the distribution by intellectual ability varies by gender, with girls more likely than boys to have an IQ $\leq 70$, and boys more likely than girls to have an IQ $>85$. $^{117}$ In children with ID, the ratio between boys and girls is closer to 2:1. ${ }^{118}$ Similar tendencies were found for adolescents and young adults. ${ }^{119}$ Studies on the cause for gender differences in ASD suggested hormonal and neurobiological factors. Fetal testosterone, for example, may explain males' vulnerability to ASD as it affects ASD genes associated with autism, and consequently alter related proteins involved in synaptic formation in the brain. Females, however, have lower levels of fetal testosterone which may have a protective effect even in the presence of genes associated with autism. ${ }^{120}$ Also, findings of brain connectivity abnormalities suggest reduced connectivity in areas related to social comprehension in males with ASD but not in females with ASD. ${ }^{121,122}$ The differences in the prevalence between boys and girls and the higher prevalence of girls with ASD in the lower range of the IQ distribution curve has long been interpreted as reflecting etiological differences. These differences, whether genetic, biological and/or environmental, have been considered as protective, so that ASD is less expressed in girls compared to boys. ${ }^{123}$ Consequently, most ASD studies tended to include participants based on higher ratio for boys over girls, or chose to include only boys or men. Hence, our knowledge of ASD characteristics in girls and women is compromised. ${ }^{48}$ However, in the last decade, there is growing evidence suggesting that the low prevalence of girls with ASD may stem at least partially from a gender bias, rather than from protective genetic or biological factors uniquely. For example, given similar levels of autistic features in children aged 2.5-4, boys were more likely to be diagnosed with autism than girls. ${ }^{124}$ Moreover, girls aged 10-12 required to show more concurrent behavioral or cognitive problems to receive a clinical diagnosis of ASD compared to boys. ${ }^{125}$ Hence, the discrepancy in ASD distribution across children is currently questioned, and researchers suggest that the low rate of girls reflects biases in perception, assessment, or diagnosis of ASD. ${ }^{126}$

The reason for the difficulty in diagnosing ASD in girls is related to "camouflage" behaviors also termed "female phenotype of autism". 127 It is suggested that girls and women may intentionally or unconsciously develop a set of strategies including masking, compensation, copying or imitation to cope or adapt to certain situations, subsequently "camouflaging" their symptoms. ${ }^{128}$ Examples of such behaviours may include imitating facial expressions of the person they are talking to, whether consciously or not, or forcing themselves to make eye contact. ${ }^{129}$, In addition, girls with ASD may show more socially competent behavior and present more communicative skills than boys by imitating social acts, interacting with others, following social scripts, and systemizing the social world. ${ }^{48}$ Girls were more likely to show an ability to integrate gestures and verbal behaviours, maintain a reciprocal conversation, and initiate but not maintain friendships compared to same-age boys. ${ }^{102}$ Female adolescents with ASD, for example, established friendships with their peers, despite their limitations in communication or difficulties in maintaining social relationships. ${ }^{130,131}$

Although camouflaging behaviors may assist girls in adjusting to the social environment at least superficially, such behaviors have serious long-term detrimental consequences that affect mental health. Maintaining these behaviors often result in late diagnosis of ASD that may prevent appropriate treatment. ${ }^{132}$ In a sample of 61 adults with ASD with no language or intellectual deficits, who were enrolled for the treatment of psychiatric comorbidities, significant differences were found between genders in age of ASD diagnosis. Women were diagnosed about 9 years later compared to men, with mean age of about 29, while men were diagnosed much earlier, with mean age of about $20 .{ }^{132}$ In another study on a group of adults with a psychiatric history, ${ }^{133}$ women usually obtained a more delayed diagnosis of ASD than men (age 26 vs age 22 respectively). Moreover, mean ADOS-2 scores of the 
Social Interaction, Communication+Social Interaction, and RRB domains were significantly lower in the group of women compared to the group of men. These findings highlight the need for developing diagnostic and screening tools that are based on the typical female phenotype and not on the typical male phenotype as well as the need for identifying camouflage autistic strategies. In continuous attempts to control and mask their symptoms, adolescent girls may show internalizing problems such as anxiety, depressive symptoms, loss of identity and self-esteem, self-injuries, or suicidal thoughts. ${ }^{128}$ Both boys and men, in contrast, appear to be at greater risk for co-occurring externalizing behaviors including ADHD, OCD and tics. ${ }^{134}$

Sex differences also characterize the expression of ADHD in individuals with ASD. In general, ADHD affects approximately $5-7 \%$ of the school age population, with a male to female ratio of about $3: 1^{135}$ or $4: 1^{136}$ in community-based samples of youth. Symptom severity is overall lower in females than in males, particularly for hyperactive-impulsive symptoms. ${ }^{137}$ Similarly to the general population, higher rate of ADHD was found for men compared to women with ASD. In a large sample of individuals with ASD, $1.01 \%$ of males showed comorbid ADHD symptoms compared to $0.43 \%$ ADHD comorbidity in women. ${ }^{138}$ In another study, boys with ASD had higher raw mean scores for ADHD traits compared to girls. However, by using standardized scores, girls with a diagnosis of ASD deviated from the sex-specific mean of ADHD traits more compared to boys signifying the importance of sex-specific norms. ${ }^{139}$ The relatively smaller number of females with ASD and ADHD compared to males as well as the less overt ADHD symptoms in females may lead to missed diagnosis and treatment.

Additionally, diagnosis of girls is further complicated by their RRBs. Girls' restricted interests tend to centre on animals, soap-operas, celebrities, pop music, fashion, horses, pets, and literature similarly to neurotypical girls, while boys' focus is on vehicles, trains, traffic lights, etc. ${ }^{48}$ There is also some evidence that girls and women show more use of imagination including fantasy, fiction, and pretend play than boys. ${ }^{48}$ In addition, it is possible that some girls have a true later onset of ASD traits than boys, or they may have subtler difficulties that take more time and greater social demands to become apparent, often, by adolescence only.

In sum, ASD is recognized more readily in girls with ID comorbidity than in those without co-occurring ID.
Girls with mild ASD symptoms and without ID, are more likely to be diagnosed at an older age compared to boys with similar symptoms. ${ }^{132}$ The greater possibility of missed or misdiagnosed ASD for this group of girls entails denial of appropriate treatment. In view of this, there is a crucial need for more studies in gender-specific clinical features, more diagnostic tools tailored to unmask autism among girls, and more early treatment that may prevent mental health issues. ${ }^{132}$ When ASD is suspected in girls, and to avoid delay or denial of crucial intervention and education, longitudinal surveillance and repeated examinations are highly recommended.

\section{ASD and Socio-Cultural Influences}

ASD can occur in any geographical region, in any linguistic or cultural group, and in all socio-economic or educational strata of society, ie, manifests as "a disorder without borders". However, its identification and treatment resources, and communities' attitude to ASD varies considerably within and between geographical regions, cultures, and Socio-Economic Status (SES) membership. Influence of demographic variables on timely and accurate identification is experienced across the globe. Especially, the economic situation of a community may influence identification and thereby the prevalence rates of ASD in children. ${ }^{140}$

Based on ASD epidemiological data that came out of northern hemisphere countries, it appeared as a disorder of those in the upper SES layer of society. However, this perception resulted from the disproportionate underdiagnoses of ASD in lower SES communities due to barriers they experienced in access to identification resources. ${ }^{141}$ For example, US studies ${ }^{29}$ found earlier diagnosis of ASD is associated with higher SES and better access to services. African American and Hispanic children tended to have later diagnosis (although the situation improved with better information dissemination), those diagnosed with ASD by age 4 tended to have coexisting ID, indicating that some African American and Hispanic children with ASD and average to above-average intelligence may not have been identified. ${ }^{29}$

A British study of 5-8 yr olds with ASD found that mothers with pre-university level education were associated with double the ASD diagnosis incidence compared to mothers with lower education, while income was not associated with this occurrence. ${ }^{142}$ A large Danish study, ${ }^{143}$ for example, showed low parental income was associated with increased ASD risk, while maternal 
education was not associated with earlier ASD diagnosis. In contrast, a US study found higher maternal education was associated with earlier ASD diagnosis. ${ }^{141}$ In the same vein, a US study in a large metropolitan southern city showed groups of children with ASD without intellectual impairment were associated with higher SES and higher maternal education. ${ }^{141}$

Autism is often shrouded in stigma in large geographical regions, and is present in all SES layers. Because of historical factors, the Arab world has only recently developed some resources for addressing children with Autism. ${ }^{144}$ However, there is increased awareness of the large number of children with ASD that are missed, leading to a growth in the number of research centres, and a campaign to urge government support of Autism research and rehabilitation institutions and raise awareness of the plight of ASD children and their families. ${ }^{144}$

Cultural factors including access to health care and different interpretation of ASD symptoms explain delayed diagnosis and treatment for different cultures and minorities. ${ }^{145,146}$ Latino and African American children with ASD, for example, are diagnosed at later ages, receive fewer treatment services, and report higher unmet service needs than white, US-born children. ${ }^{145,147}$ In addition, minority membership parents may view difficulties or delays in language and social development as a temporary stage that reflects transient immaturity rather than a developmental problem. Furthermore, they may not identify important but subtle traits for ASD such as lack of pointing, lack of imitation, lack of eye contact, and a lack of socially appropriate behavior. However, they may associate ASD with repetitive language, ritualistic behavior, and gross delays in motor skill development. ${ }^{148}$

An important question is: to what extent is the expression of ASD symptoms as defined in the DSM-5 uniform across cultures? ${ }^{149}$ In western cultures, for example, a lack of eye contact is a common deficit symptom in non-verbal communication. In the Chinese culture, children making direct eye contact with adults is considered impolite. Thus, in the Chinese cultural context, non-verbal communication difficulties characteristic for autism may manifest itself in atypical eye contact, rather than lack of eye contact. ${ }^{150}$ Also, social greetings are highly ritualized in Ethiopia and therefore "greeting others well" is reported by caregivers as something their autistic child does well. ${ }^{151}$ These examples highlight the need for further research to determine how cultural factors may affect the identification and diagnosis of autism globally. ${ }^{149}$

\section{Discussion}

This review explored the complexity and challenges in the process of an ASD differential diagnosis, and the increased risk of misdiagnosis related to weaknesses in the available diagnostic instruments. Highlighted as well is the fact that the frequent presence of comorbid conditions such as other NDDs further complicates this process, while the added burden of medical conditions present both diagnostic and treatment challenges for researchers, clinicians, and families. The review similarly expounds on the increased diagnostic difficulties encountered in early development due to the common co-occurring syndromic developmental disorders, and their shared symptomology with ASD. The comorbidity phenomenon is daunting in its results since the combined symptom severity due to the comorbidity presence is significantly greater than each of the contributing disorders, and in effect, increases with the presence of each additional comorbidity, whatever its origin.

\section{Calls for Change in ASD Diagnosis and Intervention with Children}

The differentiation problems posed by the presence of comorbidities in children and adolescents with mental health issues, for instance, is illustrated in Bickman et al study. ${ }^{152}$ A large percent (40\%) of their sample's comorbid condition test scores were practically inseparable from the suspected disorder test result making identification of a single primary diagnosis that characterizes the child's problems exceedingly difficult. Furthermore, in an editorial to a special issue, ${ }^{153}$ the authors note that, although in children 3-5 in particular, comorbidity is the rule rather than the exception, when an NDD is suspected, children will be seen by one specialist at a time despite the frequent presence of additional problems in this age group. The clinical practice of identifying early NDDs separately obscures the reality of the presence of additional disorders, and contributes to the neglect of children's complex needs when, in fact, a problem in one area often predicts future problems in the same or overlapping domains. ${ }^{154}$ The authors state that identifying and "addressing the child's complex needs together" is an urgent matter. Complex needs require comprehensive integrated intervention plans: the intervention choices and the plan effectiveness hinge on accurate identification of all contributing factors, making the researcher's ${ }^{153}$ call for change in early diagnosis practice especially poignant as it has direct impact 
on the affected children, their families, and thereby, society.

The ultimate goal in avoiding misdiagnosis and striving for an accurate ASD diagnosis, including uncovering all co-occurring problems, is to inform and facilitate effective appropriate interventions, a formidable task as clinical decisions have life-long implications for the affected children and their families. ASD interventions tend to fall along two disparate approaches, a behavioural and a developmental, each with its diverse goals and strategies. In light of the finding that numbers of individuals with ASD do not fare well with a behaviourally centred treatment approach, ${ }^{59}$ and with the available data on behavioural treatments' questionable efficacy, ${ }^{155}$ perhaps an alternative intervention paradigm may yield more striking outcomes.

In a call for change in clinical and educational practices for children with ASD that are "categorically" divided into either approach, Prizant and Mullen ${ }^{156}$ bemoan this practice:

It is unfortunate that many practitioners and agencies continue to insist on rigidly adhering to one category of practice, while claiming that other practices are ineffective and/or invalid, despite evidence to the contrary. (p.36)

In their two-part article, they propose that what is needed is "thinking developmentally", an approach that is based on theory and studies of typical and atypical human development, and which views children as active participants in learning that hinges on their stage in the developmental progression.

Accordingly, development is viewed as a process of evolving abilities in communication, language, thinking, social cognition, and other developmental domains as opposed to accumulation of discrete skills. ASD is understood as a disorder in which the expected developmental trajectory is obstructed due to underlying neurological differences. For example, a recent study showed neural circuitry and genetic mechanism differences in autism alter the severity levels in the core symptoms among individuals. ${ }^{157}$ Such differences contribute to phenotype diversity, and thereby influence their developmental trajectory. Longitudinal studies best demonstrate this concept: Canadian studies, ${ }^{158}$ for example, demonstrated dynamic trajectories with children exhibiting changes in their development and severity pathway. Other longitudinal studies, in contrast, exemplify static trajectories with stable low development and severity over time. ${ }^{159}$
In short, a "dynamic trajectory" is a desirable goal for ASD-affected children, and a developmental approach may be the path to its achievement. The essence of "developmental thinking", state Prizant and Mullen, ${ }^{156}$ is basing treatment goals on developmental stages, and practice these in natural social contexts and activities, using facilitative strategies such as "teaching, responding, and guiding flexibly in order to build on a child's intent, interests, and motivations". They encourage observing behaviours and interpreting them through the "developmental lens", an attitude and practice that is effective for individuals with ASD of any age.

Szatmari et al $^{160}$ observed in an adjunct to an ongoing Canadian longitudinal and multiregional ASD investigation (Pathways) that while studies of children with ASD tend to focus on their deficits, there is a lack in examining their positive outcomes. The researchers launched a study, using Lounds Taylor's protocol for adult ASD studies, ${ }^{161}$ to uncover the presence and prevalence of positive outcomes or "children doing well" by middle childhood, and determine the factors associated with "doing well". They followed a cohort of 272 children ( $86 \%$ boys) whose function was measured several times at ages 2-3, 4-5, and finally at mean age 10.76 .

Doing Well was operationalized as reaching established thresholds for either Proficiency (a threshold of competence to be met no matter the starting point), and/or Growth (a specific amount of improvement between two time points) in one or more of these domains: socialization, communication, independent living skills, and measures of internalizing and externalizing behaviors. In addition, measures of language, IQ, and relationship with variables such as family income, functioning, and parental coping skills were examined, as was the concurrent estimate of the association between outcomes and the ADOS (ASD presence) classification. The Proficiency metric showing percent "doing well" by domain ranged from about $20 \%$ to $49 \%$, while the Growth metric for "doing well" ranged from approximately $13 \%$ to $36 \%$. In addition, while $79 \%$ were doing well by either metric on at least one domain, $24 \%$ were doing well on 4 or 5 domains. Child attributes such as language, and family household income and functioning were significant contributors to "doing well" while cognitive function did not constitute a significant variable in either metric. Importantly, the association with the ADOS results 
clearly demonstrated that "doing well" is possible despite the continued presence of ASD. Any longitudinal studies undertaken would be wise to include studies of children on the spectrum "doing well" and determine the factors that led to the positive outcomes.

\section{Conclusions}

The challenges in identification underlined by comorbidities, gender, and socio-cultural issues raised in this review call for enhanced longitudinal studies to determine the shared characteristics of groups of children with ASD. This may facilitate avoidance of misdiagnosis, and discovery of individual functional profiles to provide a roadmap to intervention. ${ }^{2}$

The present diagnosis process and aims centre on assessment of behaviour and dysfunction, a focus that raises many ethical questions for researchers and clinicians alike as the diagnosis process, based on uncovering weaknesses, largely ignores the child's strengths. ${ }^{13}$ In addition, a recent systematic investigation ${ }^{162}$ of ASD outcome measures reported in intervention studies revealed there was no consensus on what outcomes to measure, or what measuring tools to employ for detecting changes in ASD core symptoms. Diagnostic tools, not designed to measure outcomes, used in some studies were unable to note "subtle improvements and behavioural changes" that may be important to the children and their families. The authors recommend targeting outcomes significant to families such as "quality of life", a frequently elusive goal for children and adults with ASD.

Establishing individual functional profiles would serve to highlight weaknesses, strengths, and talents, and thereby circumvent a justified ethical dilemma. Additionally, direly needed are more studies for designing diagnosis and treatment outcome instruments with a focus on autism in girls, and suitable instruments for children from socio-cultural diverse populations to facilitate accurate diagnosis, and better understanding to answer their specific needs within their cultural milieu.

In sum, this review's take-home message and future directions are the following:

- Early diagnosis tools incorporating observations of adaptive behaviours, prosocial play and associated communication with playmates, and interaction with family, caregivers, and peers; and long-term surveillance to verify diagnosis stability, and severity so as to adjust their treatment plan as needed.
- Establishing individual Functional Communication Profiles to highlight deficits and strengths, including special interests, talents, and literary preferences, to use as a guide to intervention, and the educational plan which must include long-term goals for future needs.

- Development of diagnostic tools for better identification of ASD in girls, and children from socio culturally diverse families.

- Vigilance in detecting presence of comorbidities in children with ASD is paramount. Both psychiatric and medical challenges must be addressed as these contribute to the ASD severity and impact on adaptation and participation in their social milieu.

- More studies are needed to understand children with optimal outcomes, and "doing well" so to promote the crucial factors to increase these possibilities in more children with ASD.

\section{Acknowledgments}

The authors would like to thank the anonymous reviewers for their valuable insights and comments as they enhanced the quality of this article. We would also like to thank the IALP ASD Committee for their dedication to individuals with autism via research and clinical work. Their significant contributions enrich the bank of knowledge in this complex domain.

\section{Author Contributions}

The authors contributed equally to the conception and important intellectual content: both authors made a significant contribution to the work reported in all these: the conception, the design, in drafting, revising and critically reviewing the article; gave final approval of the version to be published, have agreed on the journal to which the article has been submitted, and agree to be accountable for all aspects of the work.

\section{Disclosure}

Dr Yvette Hus is a practicing speech language pathology clinician, and a volunteer member (Deputy Chair) of the Autism Committee of the International Association of Communication Sciences and Disorders (IALP). As such, the populations of interest are children and adolescents with Autism. The authors report no conflicts of interest in this work, and neither financial nor nonfinancial interests to declare. 


\section{References}

1. Geschwind DH. Genetics of autism spectrum disorders. Trends Cogn Sci. 2011;15(9):409-416. doi:10.1016/j.tics.2011.07.003

2. Hus Y, Segal O. Functional communication profiles of children and youth with autism: a roadmap to therapeutic and educational interventions. Folia Phoniatr Logop. 2021;73:205-221. doi:10.1159/ 000510056

3. Charman T, Gotham K. Measurement issues: screening and diagnostic instruments for autism spectrum disorders - lessons from research and practice. Child Adolesc Ment Health. 2013;18:52-63. doi:10.1111/j.1475-3588.2012.00664.x

4. American Psychiatric Association. Diagnostic and Statistical Manual of Mental Disorders: DSM-5. 5thed. DSM-V; 2013. doi:10.1016/j.rasd.2014.07.021

5. Huerta M, Lord C. Diagnostic evaluation of autism spectrum disorders. Pediatr Clin North Am. 2012;59:103-111. doi:10.1016/j.pcl.2011.10.018

6. Edelson SM. Debating the role of genetics in Autism [editorial]. ARRI. 2021;34:3.

7. World Health Organization (WHO). ASD fact sheet newsroom; 2021. Available from: https://www.who.int/news-room/factsheets/detail/autism-spectrum-disorders. Accessed July 13, 2021.

8. McCarty N. Ultra-rare variants point to new autism candidate genes. Spectrum Research News; July 26, 2021. Available from: https://www.spectrumnews.org/news/ultra-rare-variants-point-tonew-autism-candidate-genes/. Accessed July 27, 2021.

9. Rylaarsdam L, Guemez-Gamboa A. Genetic causes and modifiers of autism spectrum disorders. Front Cell Neurosci. 2019;13:385. doi: $10.3389 /$ fncel.2019.00385

10. Autism Research Institute (ARI). Autism and co-occurring conditions in children. ARRI. 2021;35(1). Available from https:// www.autism.org/autism-mental-health/. Accessed July 13, 2021.

11. Vargason T, Frye R, McGuiness DL, Hahn J. Clustering of co-occurring conditions in autism spectrum disorder during early childhood: a retrospective analysis of medical claims data. Autism Res. 2019;12:1272-1285. doi:10.1002/aur.2128

12. Rolfsen E. Mental health conditions alarmingly high among children with autism. University of British Columbia Arts and Humanities News; January 19, 2021. Available From: https:// news.ubc.ca/2021/01/19/mental-health-conditions-alarminglyhigh-among-children-with-autism/. Accessed July 14, 2021.

13. Hens $\mathrm{K}$. The many meanings of autism: conceptual and ethical reflections. DMCN. 2019;61:1025-1029. doi:10.1111/dmen.14278

14. Hodges H, Fealko C, Soares N. Autism spectrum disorder: definition, epidemiology, causes, and clinical evaluation. Transl Pediatr. 2020;9(Suppl 1):S55-S65. doi:10.21037/ tp.2019.09.09

15. Soke GN, Maenner MJ, Christensen D, Kurzius-Spencer M, Schieve LA. Prevalence of co-occurring medical and behavioral conditions/symptoms among 4- and 8-year-old children with autism spectrum disorder in selected areas of the United States in 2010. J Autism Dev Disord. 2018;48(8):2663-2676. doi:10.1007/ s10803-018-3521-1

16. Meilleur -A-AS, Fombonne E. Regression of language and non-language skills in pervasive developmental disorders. J Intellect Disabil Res. 2009;53:115-124. doi:10.1111/j.13652788.2008.01134.x

17. Charman T. Developmental approaches to understanding and treating autism. Folia Phoniatr Logop. 2010;62:166-177. doi:10.1159/000314032

18. Brian AJ, Roncadin C, Duku E, et al. Emerging cognitive profiles in high-risk infants with and without autism spectrum disorder. Res Autism Spectr Disord. 2014;8:1557-1566. doi:10.1016/j. rasd.2014.07.021
19. Myers SM, Johnson CP. Management of children with autism spectrum disorders. Pediatrics. 2007;120:1162-1182. doi:10.1542/peds.2007-2362

20. Burger FL, Lang CM. Diagnoses commonly missed in childhood: long-term outcome and implications for treatment. Psychiatr Clin North Am. 1998;21(4):927-940. doi:10.1016/S0193-953X(05) 70050-2

21. Fusar-Poli L, Brondino N, Rocchetti M, et al. Diagnosing ASD in adults without ID: accuracy of the ADOS-2 and the ADI-R. J Autism Dev Disord. 2017;47:3370-3379. doi:10.1007/s10803017-3258-2

22. Volkmar FR. The importance of early intervention [editorial]. J Autism Dev Disord. 2014;44(12):2979-2980. doi:10.1007/ s10803-014-2265-9

23. Swanson AR, Warren ZE, Stone WL, Vehorn AC, Dohrmann E, Humberd Q. The diagnosis of autism in community pediatric settings: does advanced training facilitate practice change? Autism. 2014;18(5):555-561. doi:10.1177/1362361313481507

24. Devescovi R, Monasta L, Mancini A, et al. Early diagnosis and Early Start Denver Model intervention in autism spectrum disorders delivered in an Italian Public Health System service. Neuropsychiatr Dis Treat. 2016;2:1379-1384. doi:10.2147/NDT. $\mathrm{S} 106850$

25. Dawson G, Rogers S, Munson J, et al. Randomized, controlled trial of an intervention for toddlers with autism: the Early Start Denver Model. Pediatrics. 2010;125(1):e17-e23. doi:10.1542/ peds.2009-0958

26. Vivanti G, Dissanayake C; for Victorian ASELCC Team. Outcome for children receiving the Early Start Denver Model before and after 48 months. J Autism Dev Disord. 2016;46 (7):2441-2449. doi:10.1007/s10803-016-2777-6

27. Remington B, Hastings RP, Kovshoff H, et al. Early intensive behavioral intervention: outcomes for children with autism and their parents after two years. Am J Ment Retard. 2007;112(6):418-438. doi:10.1352/0895-8017(2007)112[418:EIBIOF]2.0.CO;2

28. Elder JH, Kreider CM, Brasher SN, Ansell M. Clinical impact of early diagnosis of autism on the prognosis and parent-child relationships. Psychol Res Behav Manag. 2017;10:283-292. doi:10.2147/PRBM.S117499

29. Hyman SL, Levy SE, Myers SM; for Council on Children with Disabilities, Section on Developmental and Behavioural Pediatics. Identification, evaluation, and management of children with autism spectrum disorder. Pediatrics. 2020;145(1): e20193447. doi:10.1542/peds.2019-3447

30. Anderson DK, Liang JW, Lord C. Predicting young adult outcome among more and less cognitively able individuals with autism spectrum disorders. J Child Psychol Psychiatry. 2014;55 (5):485-494. doi:10.1111/jcpp.12178

31. Wiggins LD, Baio J, Schieve L, Lee LC, Nicholas J, Rice CE. Retention of autism spectrum diagnoses by community professionals: findings from the autism and developmental disabilities monitoring network, 2000 and 2006. J Dev Behav Pediatr. 2012;33(5):387-395. doi:10.1097/DBP.0b013e3182560b2f

32. Mukaddes NM, Tutkunkardas MD, Sari O, Aydin A, Kozanoglu P. Characteristics of children who lost the diagnosis of autism: a sample from Istanbul, Turkey. Autism Res Treat. 2014;Article ID 472120:1-10. doi:10.1155/2014/472120

33. Shulman L, D'Agostino E, Lee $\mathrm{S}$, et al. When an early diagnosis of autism spectrum disorder resolves, what remains? J Child Neurol. 2019;34(7):382-386. doi:10.1177/0883073819834428

34. Mottron L. A radical change in our autism research strategy is needed: back to prototypes. Autism Res. 2021;14(10):2213-2220. doi:10.1002/aur.2494

35. Frith U. When diagnosis hampers research. [Letter to the Editor] Autism Res. 2021;1-2. doi:10.1002/aur.2578 
36. Prizant BM. On the diagnosis and misdiagnosis of autism spectrum disorder. The Cutting Edge. ASQ; 2012. Available from: https://www.asquarterly.com/. Accessed July 18, 2021.

37. Prizant BM, Wetherby AM, Rubin E, Laurent AC, Rydell PJ. The SCRETS Model. Vol 1 Assessment; Vol 2 Program Planning and Intervention. Baltimore, MD: Paul H. Brookes; 2006.

38. Randall M, Egberts KJ, Samtani A, et al. Diagnostic tests for autism spectrum disorder (ASD) in preschool children (Review). Cochrane Database Syst Rev. 2018;7:Art No: CD009044. doi:10.1002/14651858.CD009044.pub2

39. Szatmari P, Georgiades S, Duku E, et al.; for Pathways in ASD Study Team. Developmental trajectories of symptom severity and adaptive functioning in an inception cohort of preschool children with autism spectrum disorder. JAMA Psychiatry. 2015;72:276-283. doi:10.1001/jamapsychiatry.2014.2463

40. Brian JA, Zwaigenbaum L, Ip A; for Canadian Paediatric Society, Autism Spectrum Disorder Guidelines Task Force. Standards of diagnostic assessment for autism spectrum disorder [Position Statement]. Paediatr Child Health. 2019;24(7):444-451. doi:10.1093/pch/pxz117

41. Carpenter L. DSM-5 autism spectrum disorder: guidelines criteria and exemplars. DSM5; 2013. Available from: https://depts. washington.edu/dbpeds/Screening\%20Tools/DSM-5\%28ASD. Guidelines\%29Feb2013.pdf. Accessed July 24, 2021.

42. Wiggins LD, Reynolds A, Rice CE, et al. Using standardized diagnostic instruments to classify children with autism in the Study to Explore Early Development. J Autism Dev Disord. 2015;45(5):1271-1280. doi:10.1007/s10803-014-2287-3

43. National Institute for Clinical Excellence (NICE). Autism spectrum disorder in adults: diagnosis and management. NICE Clinical Guideline CG142; 2012 [Updated June, 2021]. Available from: https://www.nice.org.uk/guidance/cg142. Accessed October 23, 2021.

44. Ashwood KL, Gillan N, Horder J, et al. Predicting the diagnosis of autism in adults using the Autism-Spectrum Quotient (AQ) questionnaire. Psychol Med. 2016;46(12):2595-2604. doi:10.1017/S0033291716001082

45. Sizoo BB, Horwitz EH, Teunisse JP, et al. Predictive validity of self report questionnaires in the assessment of autism spectrum disorders in adults. Autism. 2015;19(7):842-849. doi:10.1177/ 1362361315589869

46. Fusar-Poli L, Ciancio A, Gabbiadini A, et al. Self-reported autistic traits using the AQ: a comparison between individuals with ASD, psychosis, and nonclinical controls. Brain Sci. 2020;10 (5):291. doi:10.3390/brainsci10050291

47. Richler J, Huerta M, Bishop SL, Lord C. Developmental trajectories of restricted and repetitive behaviors and interests in children with autism spectrum disorders. Dev Psychopathol. 2010;22 (1):55-69. doi:10.1017/S0954579409990265

48. Lai MC, Lombardo MV, Auyeung B, Chakrabarti B, BaronCohen S. Sex/gender differences and autism: setting the scene for future research. $J$ Am Acad Child Adolesc Psychiatry. 2015;54:11-24. doi:10.1016/j.jaac.2014.10.003

49. Gulisano M, Barone R, Alaimo S, et al. Disentangling restrictive and repetitive behaviors and social impairments in children and adolescents with Gilles de la Tourette syndrome and autism spectrum disorder. Brain Sci. 2020;10(5):308. doi:10.3390/ brainsci10050308

50. Global Genes Allies in Rare Diseases. Tourette syndrome. Available from: https://globalgenes.org/disease/tourettesyndrome. Accessed October 14, 2021.

51. Ueda K, Black KJ. A comprehensive review of Tic disorders in children. J Clin Med. 2021;10(11):2479. doi:10.3390/jcm10112479

52. Norbury CF. Practitioner review: social (pragmatic) communication disorder conceptualization, evidence and clinical implications. J Child Psychol Psychiatry. 2014;55(3):204-216. doi:10.1111/jcpp.12154
53. Brukner-Wertman Y, Laor N, Golan O. Social (pragmatic) communication disorder and its relation to the autism spectrum: dilemmas arising from the DSM-5 classification. J Autism Dev Disord. 2016;46:2821-2829. doi:10.1007/s10803-016-2814-5

54. Mandy W, Wang A, Lee I, Skuse D. Evaluating social (pragmatic) communication disorder. JCPP Adv. 2017;58(10):1166-1175. doi:10.1111/jcpp. 12785

55. Visser S, Tops W. Social pragmatic communication disorder and autism spectrum disorder: two of a kind? A narrative review. Ann Psychiatry Ment Health. 2017;5(6):1121. doi:10.33588/ rn.4412.2006434

56. Muskens JB, Velders FP, Staal WG. Medical comorbidities in children and adolescents with autism spectrum disorders and attention deficit hyperactivity disorders: a systematic review. Eur Child Adolesc Psychiatry. 2017;26:1093-1103. doi:10.1007/ s00787-017-1020-0

57. Aldinger KA, Lane CJ, Veenstra-VanderWeele J, Levitt P. Patterns of risk for multiple co-occurring medical conditions replicate across distinct cohorts of children with autism spectrum disorder. Autism Res. 2015;8:771-781. doi:10.1002/aur.1492

58. Ng QX, Loke W, Venkatanarayanan N, Lim DY, Soh AYS, Yeo WS. A systematic review of the role of prebiotics and probiotics in autism spectrum disorders. Medicina (Kaunas). 2019;55(5):129. doi:10.3390/medicina55050129

59. Feroe AG, Uppal N, Gutiérrez-Sacristán A, et al. Medication use in the management of comorbidities among individuals with autism spectrum disorder from a large nationwide insurance database. JAMA Pediatr. 2021;175(9):957. doi:10.1001/ jamapediatrics.2021.1329

60. Rittner B. Diagnosis du jour? Considering orthorexia nervosa. social work synergy, the blog of the UB school of social work; May 7, 2014. Available from: https://socialworksynergy.org/cate gory/barbara-rittner/. Accessed October 18, 2021.

61. Hong MP, Erickson CA. Investigational drugs in early-stage clinical trials for autism spectrum disorder. Expert Opin Investig Drugs. 2019;28(8):709-718. doi:10.1080/13543784.2019.1 649656

62. Davidovitch M, Hemo B, Manning-Courtney P, Fombonne E. Prevalence and incidence of autism spectrum disorder in an Israeli population. J Autism Dev Disord. 2013;43(4):785-793. doi:10.1007/s10803-012-1611-z

63. Staikova E, Gomes H, Tartter V, McCabe A, Halperin JM. Pragmatic deficits and social impairment in children with ADHD. J Child Psychol Psychiatry. 2013;54(12):1275-1283. doi:10.1111/jcpp. 12082

64. Sumner E, Leonard HC, Hill EL. Comparing attention to socially-relevant stimuli in autism spectrum disorder and developmental coordination disorder. J Abnorm Child Psychol. 2018;46:1717-1729. doi:10.1007/s10802-017-0393-3

65. Kleinman JM, Robins DL, Ventola PE, et al. The modified checklist for autism in Toddlers: a follow-up study investigating the early detection of autism spectrum disorders. J Autism Dev Disord. 2008;38:827-839. doi:10.1007/s10803-007-0450-916

66. Guthrie W, Swineford LB, Nottke C, Wetherby AM. Early diagnosis of autism spectrum disorder: stability and change in clinical diagnosis and symptom presentation. J Child Psychol Psychiatry. 2013;54(5):582-590. doi:10.1111/jcpp. 12008

67. Leitner Y. The co-occurrence of autism and attention deficit hyperactivity disorder in children - what do we know? Front Hum Neurosci. 2014;29(8):268. doi:10.3389/fnhum.2014.00268

68. Simonoff E, Pickles A, Charman T, Chandler S, Loucas T, Baird G. Psychiatric disorders in children with autism spectrum disorders: prevalence, comorbidity, and associated factors in a population-derived sample. $J$ Am Acad Child Adolesc Psychiatry. 2008;47(8):921-929. doi:10.1097/CHI.0b013 e318179964f 
69. Zablotsky B, Bramlett MD, Blumberg SJ. The co-occurrence of autism spectrum disorder in children with ADHD. J Atten Disord. 2020;24(1):94-103. doi:10.1177/1087054717713638

70. Tek S, Mesite L, Fein D, et al. Longitudinal analyses of expressive language development reveal two distinct language profiles among young children with autism spectrum disorders. J Autism Dev Disord. 2014;44:75-89. doi:10.1007/s10803-013-1853-4

71. Groom MJ, Kochhar P, Hamilton A, et al. Atypical processing of gaze cues and faces explains comorbidity between Autism Spectrum Disorder (ASD) and Attention Deficit/Hyperactivity Disorder (ADHD). J Autism Dev Disord. 2017;47:1496-1509. doi:10.1007/s10803017-3078-4

72. Tye C, Mercure E, Ashwood KL, et al. Neurophysiological responses to faces and gaze direction differentiate children with ASD, ADHD and ASD plus ADHD. Dev Cogn Neurosci. 2013;5:71-85. doi:10.1016/j.den.2013.01.001

73. Vandewouw MM, Choi E, Hammill C, et al. Emotional face processing across neurodevelopmental disorders: a dynamic faces study in children with autism spectrum disorder, attention deficit hyperactivity disorder and obsessive-compulsive disorder. Transl Psychiatry. 2020;10:375. doi:10.1038/s41398-020-01063-2

74. Dawson G, Webb SJ, McPartland J. Understanding the nature of face processing impairment in autism: insights from behavioral and electrophysiological studies. Dev Neuropsychol. 2005;27 (3):403-424. doi:10.1207/s15326942dn2703_6

75. Wolraich M, Brown L, Brown RT, et al.; Subcommittee on attention-deficit/hyperactivity disorder; steering committee on quality improvement and management. ADHD: clinical practice guideline for the diagnosis, evaluation, and treatment of attention deficit/hyperactivity disorder in children and adolescents. Pediatrics. 2011;128(5):1007-1022. doi:10.1542/peds.2011-2654

76. Geurts M, Embrechts M. Language profiles in ASD, SLI and ADHD. J Autism Dev Disord. 2008;38:1931-1943. doi:10.1007/ s10803-008-0587-1

77. Georgiou N, Spanoudis G. Developmental language disorder and autism: commonalities and differences on Language. Brain Sci. 2021;589:1-29. doi:10.3390/brainsci11050589

78. Andrés-Roqueta CA, Katsos N. A distinction between linguistic and social pragmatics helps the precise characterization of pragmatic challenges in children with autism spectrum disorders and developmental language disorder. J Speech Lang Hear Res. 2020;63:1494-1508. doi:10.1044/2020_JSLHR-19-00263

79. Baird G, Simonoff E, Pickles A, et al. Prevalence of disorders of the autism spectrum in a population cohort of children in South Thames: the Special Needs and Autism Project (SNAP). Lancet. 2006;368:210-215. doi:10.1016/S0140-6736(06)69041-7

80. Levy SE, Giarelli E, Lee LC, et al. Autism spectrum disorder and co-occurring developmental, psychiatric, and medical conditions among children in multiple populations of the United States. $J$ Dev Behav Pediatr. 2010;31:267-275. doi:10.1097/ DBP.0b013e3181d5d03b

81. Jo'nsdo'ttir SL, Saemundsen E, Antonsdo'ttir IS, Sigurdardo 'ttir S, O'lason D. Children diagnosed with autism spectrum disorder before or after the age of 6 years. Res Autism Spectr Disord. 2011;5:175-184. doi:10.1016/j.rasd.2010.03.007

82. Kjelgaard MM, Tager-Flusberg H. An investigation of language impairment in autism: implications for genetic subgroups. Lang Cogn Process. 2001;16:287-308. doi:10.1080/016909600 42000058

83. Roberts JA, Rice ML, Tager-Flusberg H. Tense marking in children with autism. Appl Psycholinguist. 2004;25:429-448. doi:10.1017/S0142716404001201

84. Paul R, Chawarska K, Volkmar F. Differentiating ASD from DLD in toddlers. Perspect Lang Learn Educ. 2008;15:101-111. doi:10.1044/1le15.3.101
85. Liddle B, Nettle D. Higher-order theory of mind and social competence in school-age children. J Cult Evol Psychol. 2006;4 (3-4):231-246. doi:10.1556/JCEP.4.2006.3-4.3

86. Lloyd-Esenkaya V, Russell AJ, Clair MCS. What are the peer interaction strengths and difficulties in children with developmental language disorder? A systematic review. Int J Environ Res Public Health. 2020;17(9):3140. doi:10.3390/ijerph17093140

87. Wolfberg P, DeWitt M, Young GS, et al. Integrated play groups: promoting symbolic play and social engagement with typical peers in children with ASD across settings. $J$ Autism Dev Disord. 2015;45:830-845. doi:10.1007/s10803-014-2245-0

88. Miller HL, Sherrod GM, Mauk JE, Fears NE, Hynan LS, Tamplain PM. Shared features or co-occurrence? Evaluating symptoms of developmental coordination disorder in children and adolescents with autism spectrum disorder. J Autism Dev Disord. 2021;51 (10):3443-3455. doi:10.1007/s10803-020-04766-z

89. Bhat AN. Is motor impairment in autism spectrum disorder distinct from developmental coordination disorder? A report from the SPARK study. PhysTher. 2020;100(4):633-644. doi:10.1093/ $\mathrm{ptj} / \mathrm{pzz} 190$

90. Kanner L. Autistic disturbances of affective contact. Acta Paedopsychiatr. 1968;35(4):100-136.

91. Dziuk MA, Gidley Larson JC, Apostu A, Mahone EM, Denckla MB, Mostofsky SH. Dyspraxia in autism: association with motor, social, and communicative deficits. DMCN. 2007;49:734-739. doi:10.1111/j.1469-8749.2007.00734.x

92. Downey R, Rapport MJ. Motor activity in children with autism: a review of current literature. Pediatr Phys Ther. 2012;24 (1):2-20. doi:10.1097/PEP.0b013e31823db95f

93. Fournier KA, Hass CJ, Naik SK, Lodha N, Cauraugh JH. Motor coordination in autism spectrum disorders: a synthesis and meta-analysis. J Autism Dev Disord. 2010;40:1227-1240. doi:10.1007/s10803-010-0981-3

94. Bishop S, Gahagan S, Lord C. Re-examining the core features of autism: a comparison of autism spectrum disorder and fetal alcohol spectrum disorder. J Child Psychol Psychiatry. 2007;48 (11):1111-1121. doi:10.1111/j.1469-7610.2007.01782.x75

95. Grzadzinski R, Dick C, Lord C, Bishop S. Parent-reported and clinician observed autism spectrum disorder (ASD) symptoms in children with attention deficit/hyperactivity disorder (ADHD): implications for practice under DSM-5. Mol Autism. 2016;7:7. doi:10.1186/s13229-016-0072-1

96. Caçola P, Miller HL, Ossom Williamson P. Behavioral comparisons in autism spectrum disorder and developmental coordination disorder: a systematic literature review. Res Autism Spectr Disord. 2017;38:6-18. doi:10.1016/j.rasd.2017.03.004

97. Srivastava AK, Schwartz CE. Intellectual disability and autism spectrum disorders: causal genes and molecular mechanisms. Neurosci Biobehav Rev. 2014;46(Pt 2):161-174. doi:10.1016/j. neubiorev.2014.02.015

98. Matson JL, Dempsey T, Fodstad JC. The effect of autism spectrum disorders on adaptive independent living skills in adults with severe intellectual disability. Res Dev Disabil. 2009;30 (6):1203-1211. doi:10.1016/j.ridd.2009.04.001

99. Artigas-Pallarés J, Rigau-Ratera E, García-Nonell C. The relation between borderline intellectual capacity and neurodevelopmental disorders [abstract]. Rev Neurol. 2007;44(12):739-744. PMID: 17583867.

100. La Malfa GP, Lassi S, Bertelli M, Salvini R, Placidi GF. Autism and intellectual disability: a study of prevalence on a sample of the Italian population. J Intellect Disabil Res. 2004;48:262-267. doi:10.1111/j.1365-2788.2003.00567.x

101. Lemcke S, Juul S, Parner ET, et al. Early signs of autism in toddlers: a follow-up study in the Danish National Birth Cohort. J Autism Dev Disord. 2013;43:2366-2375. doi:10.1007/s10803013-1785-z 
102. Armstrong ES, Yokel A. Language outcomes for preverbal toddlers with autism. Stud Lang Lit. 2012;4:1-7. doi:10.3968/j. sll.1923156320120403.3528

103. Brignell A, Morgan A, Woolfenden S, et al. A systematic review and meta-analysis of the prognosis of language outcomes for individuals with autism spectrum disorder. Autism Dev Lang Impair. 2018;3:1-19. doi:10.1177/2396941518767610

104. Polyak A, Kubina RM, Girirajan S. Comorbidity of intellectual disability confounds ascertainment of autism: implications for genetic diagnosis. Am J Med Genet B Neuropsychiatr Genet. 2015;168(7):600-608. doi:10.1002/ajmg.b.32338

105. Farooqi S, Jabeen A. Child with intellectual disability misdiagnosed as autism: a case study. J Pak Med Assoc. 2021;71 (8):2077-2079. doi:10.47391/JPMA.365

106. Duncan AW, Bishop SL. Understanding the gap between cognitive abilities and daily living skills in adolescents with autism spectrum disorders with average intelligence. Autism. 2015;19 (1):64-72. doi:10.1177/1362361313510068

107. Kurki MI, Saarentaus E, Pietilainen O, et al. Contribution of rare and common variants to intellectual disability in a sub-isolate of Northern Finland. Nat Commun. 2019;10(1):410. doi:10.1101/ 332023

108. Fombonne E. Epidemiological trends in rates of autism. Mol Psychiatry. 2002;7(Suppl 2):S4-6. doi:10.1038/sj.mp.4001162

109. Ben-Itzchak E, Watson LR, Zachor DA. Cognitive ability is associated with different outcome trajectories in autism spectrum disorders. J Autism Dev Disord. 2014;44(9):2221-2229. doi:10.1007/s10803-014-2091-0

110. Contaldo A, Colombi C, Pierotti C, Masoni P, Muratori F. Outcomes and moderators 55 of Early Start Denver Model intervention in young children with autism spectrum disorder 56 delivered in a mixed individual and group setting. Autism. 2020;24(3):718-729. doi:10.1177/1362361319888344

111. Zachor DA, Ben-Itzchak E. Variables affecting outcome of early intervention in autism spectrum disorder. Pediatr Neurol. 2017;15:129-133. doi:10.1055/s-0037-1601444

112. Mahmood SN, Jabeen A. Silence misdiagnosed: mutism, autism or submissiveness? Diagnostic bias in collectivistic culture. Khyber Med Univ J. 2018;10(3):159-162.

113. Driessen J, Blom JD, Muris P, et al. Anxiety in children with selective Mutism: a meta-analysis. Child Psychiatry Hum Dev. 2020;51:330-341. doi:10.1007/s10578-019-00933-1

114. Muris P, Ollendick TH. Selective Mutism and is relations to social anxiety disorder and autism spectrum disorder. Clin Child Fam Psychol Rev. 2021;24(2):294-325. doi:10.1007/s10567-02000342-0

115. Steffenburg H, Steffenburg S, Gillberg C, Billstedt E. Children with autism spectrum disorders and selective mutism. Neuropsychiatr Dis Treat. 2018;14:1163-1169. doi:10.2147/ NDT.S154966

116. Werling DM, Geschwind DH. Sex differences in autism spectrum disorders. Curr Opin Neurol. 2013;26(2):146-153. doi:10.1097/ WCO.0b013e32835ee548

117. Baio J, Wiggins L, Christensen DL, et al. Prevalence of autism spectrum disorder among children aged 8 years: autism and developmental disabilities monitoring network, 11 sites, United States, 2014. MMWR Surveill Summ. 2018;67(SS-6):1-23. doi:10.15585/mmwr.ss6706a1

118. Yeargin-Allsopp M, Rice C, Karapurkar T, Doernberg N, Boyle C, Murphy C. Prevalence of autism in a US metropolitan area. JAMA. 2003;289(1):49-55. doi:10.1001/jama.289.1.49

119. Loomes R, Hull L, Mandy WPL. What is the male-to-female ratio in autism spectrum disorder? A systematic review and meta-analysis. JAACAP. 2017;56(6):466-474. doi:10.1016/j. jaac.2017.03.013
120. Ferri SL, Abel T, Brodkin ES. Sex differences in autism spectrum disorder: a review. Curr Psychiatry Rep. 2018;20(2):9. doi:10.1007/s11920-018-0874-2

121. Kirkovski M, Enticott PG, Hughes ME, Rossell SL, Fitzgerald PB. Atypical neural activity in males but not females with autism spectrum disorder. $J$ Autism Dev Disord. 2016;46:954-963. doi:10.1007/s10803-015-2639-7

122. Zeestraten EA, Gudbrandsen MC, Daly E, et al. Sex differences in frontal lobe connectivity in adults with autism spectrum conditions. Transl Psychiatry. 2017;7:e1090. doi:10.1038/ tp.2017.9

123. Wing L, Lord C. Some questions on sex differences. J Autism Dev Disord. 1984;14:211-214. doi:10.1007/BF02409664

124. Russell G, Steer C, Golding J. Social and demographic factors that influence the diagnosis of autistic spectrum disorders. Soc Psychiatry Psychiatr Epidemiol. 2011;46(12):1283-1293. doi:10.1007/s00127-010-0294-z

125. Dworzynski K, Ronald A, Bolton P, Happé F. How different are girls and boys above and below the diagnostic threshold for autism spectrum disorders? $J$ Am Acad Child Adolesc Psychiatry. 2012;51(8):788-797. doi:10.1016/j.jaac.2012.05.018

126. Kirkovski M, Enticott PG, Fitzgerald PB. A review of the role of female gender in autism spectrum disorders. J Autism Dev Disord. 2013;45(11):2584-2603. doi:10.1007/s10803-013-1811-1

127. Schuck RK, Flores RE, Fung LK. Brief report: sex/gender differences in symptomology and camouflaging in adults with autism spectrum disorder. J Autism Dev Disord. 2019;49(6):2597-2604. doi:10.1007/s10803-019-03998-y

128. Tubío-Fungueiriño M, Cruz S, Sampaio A, Carracedo A, Fernández-Prieto M. Social camouflaging in feMCA with autism spectrum disorder: a systematic review. $J$ Autism Dev Disord. 2021;51(7):2190-2199. doi:10.1007/s10803-020-04695-x

129. Hull L, Petrides KV, Mandy W. The female autism phenotype and camouflaging: a narrative review. Rev J Autism Dev Disord. 2020;7:306-317. doi:10.1007/s40489-020-00197-9

130. Hiller RM, Young R, Weber N. Sex differences in autism spectrum disorder based on DSM-5 criteria: evidence from clinician and teacher reporting. J Abnorm Child Psychol. 2014;42 (8):1381-1393. doi:10.1007/s10802-014-9881-x

131. Cook A, Ogden J, Winstone N. Friendship motivations, challenges and the role of masking for girls with autism in contrasting school settings. Eur J Spec Needs Educ. 2018;33(3):302-315. doi:10.1080/08856257.2017.1312797

132. Gesi C, Migliarese G, Torriero S, et al. Gender differences in misdiagnosis and delayed diagnosis among adults with autism spectrum disorder with no language or intellectual disability. Brain Sci. 2021;11(7):912. doi:10.3390/brainsci11070912

133. Fusar-Poli L, Brondino N, Politi P, et al. Missed diagnoses and misdiagnoses of adults with autism spectrum disorder. Eur Arch Psychiatry Clin Neurosci. 2020. doi:10.1007/s00406-02001189-w

134. Rynkiewicz A, Łucka I. Autism spectrum disorder (ASD) in girls. Co-occurring psychopathology. Sex differences in clinical manifestation. Psychiatr Pol. 2018;52(4):629-639. doi:10.12740/PP/OnlineFirst/58837

135. Willcutt EG. The prevalence of DSM-IV attention deficit/hyperactivity disorder: a meta-analytic review. Neurotherapeutics. 2012;9:490-499. doi:10.1007/s13311-012-0135-8

136. Cantwell DP. Attention deficit disorder: a review of the past 10 years. J Am Acad Child Adolesc Psychiatry. 1996;35:978-987. doi:10.1097/00004583-199608000-00008

137. Young S, Adamo N, Ásgeirsdóttir BB, et al. Females with ADHD: an expert consensus statement taking a lifespan approach providing guidance for the identification and treatment of attention-deficit/ hyperactivity disorder in girls and women. BMC Psychiatry. 2020;20:404. doi:10.1186/s12888-020-02707-9 
138. Ghirardi L, Brikell I, Kuja-Halkola R, et al. The familial co-aggregation of ASD and ADHD: a register-based cohort study. Mol Psychiatry. 2018;23:257-262. doi:10.1038/mp.2017.17

139. Lundström S, Mårland C, Kuja-Halkola R, et al. Assessing autism in females: the importance of a sex-specific comparison. Psychiatry Res. 2019;282:112566. doi:10.1016/j.psychres.2 019.112566

140. Nowell KP, Brewton CM, Allain E, Mire SS. The influence of demographic factors on the identification of autism spectrum disorder: a review and call for research. Rev $J$ Autism Dev Disord. 2015;2:300-309. doi:10.1007/s40489-015-0053-x

141. Accordino RE, Green IW, Angela Diaz A. Autism spectrum disorder in lower socioeconomic communities: viewpoint. Ann Glob Health. 2017;83(5-6):753-755. doi:10.1016/j.aogh.20 17.10.018

142. Kelly B, Williams S, Collins S, et al. The association between socioeconomic status and autism diagnosis in the United Kingdom for children aged 5-8 years of age: findings from the born in Bradford cohort. Autism. 2019;23(1):131-140. doi: $10.1177 / 1362361317733182$

143. Larsson HJ, Eaton WW, Madsen KM, et al. Risk factors for autism: perinatal factors, parental psychiatric history, and socioeconomic status. Am J Epidemiol. 2005;161(10):916-925. doi:10.1093/aje/kwi123

144. Hassan A. Arab views on Autism. In: Volkmar F, editor. Encyclopedia of Autism Spectrum Disorders. New York: Springer; 2012:2-5. doi:10.1007/978-1-4614-6435-8_1023091

145. Burkett K, Morris E, Manning-Courtney $P$, et al. African American families on autism diagnosis and treatment: the influence of culture. J Autism Dev Disord. 2015;45:3244-3254. doi:10.1007/s10803-015-2482-x

146. Kang-Yi CD, Grinker RR, Mandell DS. Korean culture and autism spectrum disorders. $J$ Autism Dev Disord. 2013;43 (3):503-520. doi:10.1007/s10803-012-1570-4

147. Angell AM, Empey A, Zuckerman KE. A review of diagnosis and service disparities among children with autism from racial and ethnic minority groups in the United States. In: Hodapp RM, Fidler DJ, editors. International Review of Research in Developmental Disabilities. Vol. 55. Massachusetts: Academic Press; 2018:145-180.

148. Ennis-Cole D, Durodoye BA, Harris HL. The impact of culture on autism diagnosis and treatment: considerations for counselors and other professionals. Fam J Alex Va. 2013;21(3):279-287. doi:10.1177/1066480713476834

149. de Leeuw A, Happé FGE, Hoekstra RAA. Conceptual framework for understanding the cultural and contextual factors on autism across the globe. Autism Res. 2020;13(7):1029-1050. doi:10.100 2/aur.2276

150. Zhang J, Wheeler J, Richey D. Cultural validity in assessment instruments for children with autism from a Chinese cultural perspective. Int J Spec Educ. 2006;21:109-114.
151. Hoekstra RA, Bayouh FG, Gebru BT, et al. The face of autism in Ethiopia: the expression, recognition, reporting and interpretation of autism symptoms in the Ethiopian context. Oral Presentation at: Annual Meeting of the International Society for Autism Research (INSAR); May 9; 2018; Rotterdam, Netherlands.

152. Bickman L, Wighton LG, Lambert EW, Karver MS, Steding L. Problems in using diagnosis in child and adolescent mental health services research. J Methods Meas Soc Sci. 2012;3(1):1-26. doi:10.2458/jmm.v3i1.16110

153. Gillberg C, Fernell E, Minnis H. Early symptomatic syndromes eliciting neurodevelopmental clinical examinations [editorial]. Sci World J. 2013;2013:1-2. doi:10.1155/2013/710570

154. Gillberg C. The ESSENCE in child psychiatry: early symptomatic syndromes eliciting neurodevelopmental clinical examinations. Res Dev Disabil. 2010;31(6):1543-1551. doi:10.1016/j. ridd.2010.06.002

155. Mottron L. Should we change targets and methods of early intervention in autism, in favor of a strengths-based education? Eur Child Adolesc Psychiatry. 2017;26:815-825. doi:10.1007/s00787017-0955-52017

156. Prizant BM, Mullen E. Straight Talk about Autism: Thinking developmentally. ASQ 18 (Pt 1) \& ASQ 19 (Pt 2). 2012. Available at:https://www.asquarterly.com/. Accessed August 9, 2021.

157. Bertelsen N, Landi I, Bethlehem RAI, et al. Imbalanced social-communicative and restricted repetitive behavior subtypes of autism spectrum disorder exhibit different neural circuitry. Commun Biol. 2021;4(1):574. doi:10.1038/s42003-021-02015-2

158. Georgiades S, Bishop SL, Frazier T. Longitudinal research in autism: introducing the concept of 'chronogeneity' [editorial]. J Child Psychol Psychiatry. 2017;5(58):634-636. doi:10.1111/ jcpp. 12690

159. Baghdadli A, Rattaz C, Michelon C, Pernon E, Munir K. Fifteenyear prospective follow-up study of adult outcomes of autism spectrum disorders among children attending centers in five regional departments in France: the EpiTED Cohort. J Autism Dev Disorder. 2019;49(6):2243-2256. doi:10.1007/s10803-01903901-9

160. Szatmari P, Tombeau Cost K, Duku E, et al. Association of child and family attributes with outcomes in children with autism. JAMA Netw Open. 2021;4(3):e212530. doi:10.1001/jamanet workopen.2021.2530

161. Lounds Taylor J. When is good outcome actually good? [Editorial]. AUTISM. 2017;21(8):918-919. doi:10.1177/13623 61317728821

162. Provenzani U, Fusar-Poli L, Brondino N, et al. What are we targeting when we treat autism spectrum disorder? A systematic review of 406 clinical trials. Autism. 2020;24(2):274-284 doi: $10.1177 / 1362361319854641$
Neuropsychiatric Disease and Treatment

\section{Publish your work in this journal}

Neuropsychiatric Disease and Treatment is an international, peerreviewed journal of clinical therapeutics and pharmacology focusing on concise rapid reporting of clinical or pre-clinical studies on a range of neuropsychiatric and neurological disorders. This journal is indexed on PubMed Central, the 'PsycINFO' database and CAS, and is the official journal of The International Neuropsychiatric Association (INA). The manuscript management system is completely online and includes a very quick and fair peer-review system, which is all easy to use. Visit http://www.dovepress.com/testimonials.php to read real quotes from published authors. 